\title{
DETERMINAÇÃO SIMULTÂNEA DE COBALTO E NÍQUEL
}

Patrícia Alexandra Antunes, Susete Trazzi Breviglieri, Gilberto Orivaldo Chierice

Departamento de Química e Física Molecular - Instituto de Química de São Carlos/USP - Av. Dr. Carlos Botelho, 1465 - CP 780 13560-970 - São Carlos - SP

Éder Tadeu Gomes Cavalheiro

Departamento de Química - Universidade Federal de São Carlos - Via Washington Luís, Km 235 - CP 676 - $13565-905$ São Carlos - SP

Recebido em 19/2/97; aceito em 18/6/97

\begin{abstract}
SIMULTANEOUS DETERMINATIONS OF NICKEL AND COBALT - A REVIEW. The simultaneous determination of cobalt and nickel is a classical analytical problem. A great number of reagents associated with several techniques of analysis have been applied to achieve this determination. In this review 117 references, which appeared between 1996-1980, are described and classified according to the technique applied.
\end{abstract}

Keywords: nickel; cobalt; simultaneous determination.

\section{INTRODUÇÃO}

A determinação simultânea de Cobalto e Níquel é um problema clássico na Química Analítica. Assim sendo, um grande número de técnicas envolvendo diferentes reagentes e estratégias são freqüentemente propostas para essa finalidade.

Recentemente, aproveitando a diferença de coloração de soluções contendo $\mathrm{Ni}^{2+}$ e $\mathrm{Co}^{2+}$ em presença de dietanolditiocarbamato, foi proposto um método espectrofotométrico direto e simultâneo para determinação destes cátions. Determinou-se a absorbância em diferentes comprimentos de onda, com a vantagem de que a determinação direta dispensou a necessidade de tratamento matemático posterior ${ }^{1}$.

Um levantamento bibliográfico foi realizado para auxiliar naquele trabalho junto ao banco de dados em CD-ROM, "Analytical Abstracts", no período entre 1996-1983, utilizando a seguinte expressão de busca: "nickel and cobalt and simultaneous". Esta base de dados só apresenta trabalhos completos publicados por revistas especializadas em química analítica, não incluindo artigos apresentados em anais de reuniões científicas nacionais ou internacionais, bem como periódicos de caráter genérico.

Nestas condições foram localizadas 117 citações sobre o assunto, neste período, sob aquela expressão de busca, na base de dados consultada.

O elevado número de referências encontrado neste levantamento motivou a preparação desta revisão, a qual pretende auxiliar aos que trabalham neste tipo de determinação, apresentando metodologias utilizadas e resultados obtidos pelos diversos autores. Assim os trabalhos foram classificados por técnicas empregadas nas determinações em cromatografia, eletroanalítica, espectrometria, FIA e métodos mistos. A figura 1 apresenta a distribuição percentual dos artigos aqui selecionados, em relação a cada técnica.

\section{CROMATOGRAFIA}

Um procedimento cromatográfico baseado na separação de complexos de PAR, por CLAE, foi proposto por Dolezal e Sommer ${ }^{2}$, para a determinação simultânea de Co, Ni e Fe utilizando dois procedimentos. O primeiro foi de preparar os complexos à parte, após o que estes foram adicionadoa a uma précoluna de Separon SGX-RPS (2 $\mathrm{cm}$ x 9mm). Os complexos foram eluídos em contra-corrente usando-se metanol. $\mathrm{O}$ eluato foi analisado, por CLAE, em uma coluna de Separon SGX-RPS

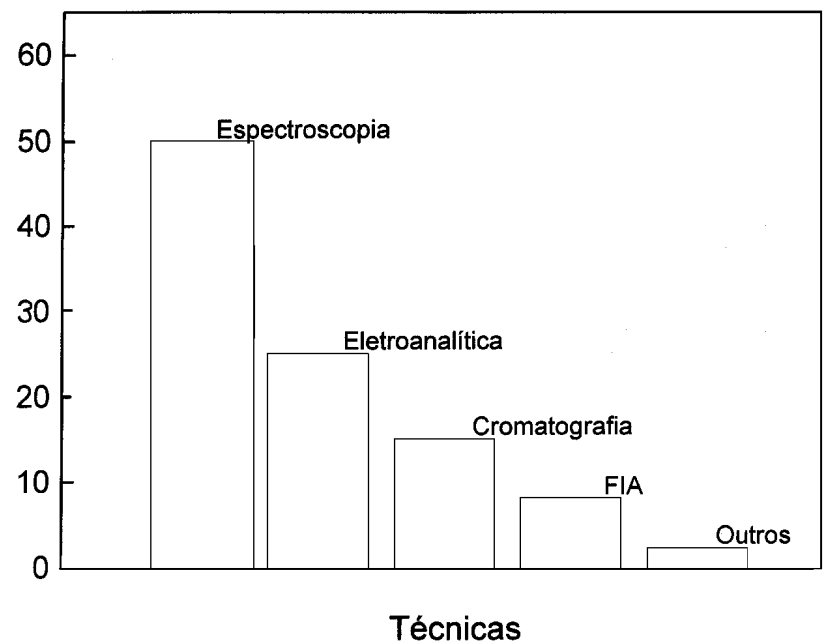

Figura 1. Distribuição percentual dos trabalhos citados em relação às técnicas empregadas.

(25 cm x $4 \mathrm{~mm}$ ), utilizando uma fase móvel de hexametilenotetraanina e brometo de hexadeciltrimetilamônio, com detecção em comprimentos de onda variáveis. No procedimento em linha uma coluna de vidro de Separon SGX, foi colocada no topo da coluna CLAE. Os metais foram inseridos juntamente com PAR em $\mathrm{pH}=6$, formando os complexos. A eluição e detecção foram realizadas como descrito acima. As figuras de mérito para cada procedimento são descritas pelos autores.

Gonzalez-Rodrigues et $\mathrm{al}^{3}$ apresentaram estudos utilizando uma solução de ácido dietilditiocarbâmico em solução de etanol-água (50\%), em pH 10, utilizada para complexação dos metais, estes foram extraídos com $10 \mathrm{~mL}$ de éter etílico. Após este procedimento uma fração da fase orgânica foi diluída com metanol. Uma alíquota desta solução resultante foi finalmente analisada em uma coluna Waters Nova Pak C18 (15 cm x 0,39 $\mathrm{cm}$ de diâmetro interno), usando como fase estacionária sílica amorfa ligada a dimetiloctadecilsilil. A fase móvel utilizada foi o metanol aquoso $(75 \%)$ e o comprimento de onda de detecção regulado em $260 \mathrm{~nm}$. As curvas analíticas obtidas pelos autores se mostraram lineares entre 0,01-1 ppm de Co e 
0,1-5 ppm de Ni com limite de detecção em 5 e 50 ppb respectivamente. Dentre os cátions testados, $\mathrm{Se}(\mathrm{IV}), \mathrm{Ba}, \mathrm{Be}, \mathrm{Ca}, \mathrm{Sr}$, $\mathrm{K}$, e Ag se mostraram interferentes ao nível de $10 \mathrm{ppm}$, que foi a concentração de teste utilizada.

Os mesmos autores ${ }^{4}$ realizaram ainda um segundo estudo sobre a determinação de metais incluindo neste trabalho a detecção de $\mathrm{Cu}, \mathrm{Co}$ e Ni. Detalhes experimentais são idênticos ao anterior, quanto ao procedimento de extração, reagente, coluna, detector, etc. Os resultados obtidos geraram gráficos lineares para 2,5-50 (Cu), 2,5-50 (Co), 25-500 ppm (Ni). Os limites de detecção foram respectivamente $30 ; 5$ e 50 ppb, com RSD entre 3,15-4,61\%. Interferiram ao nível de $50 \mathrm{ppm} \mathrm{Al}$, $\mathrm{Fe}, \mathrm{Cd}, \mathrm{Sr}$, e $\mathrm{Ba}$. Segundo os autores o método é útil para a determinação dos metais em ligas metálicas.

A aplicação de coluna Waters Delta Pak RP-18 na determinação simultânea de metais foi relatada por Sturaro et $\mathrm{al}^{5}$. A fase móvel utilizada foi uma mistura de acetonitrila, octasulfato de sódio e hidrogeno-tartarato de sódio, em $\mathrm{pH}=3,65$. A detecção foi efetuada espectrofotometricamente utilizando-se arranjo linear de diodos. Derivatização com PAR, em linha com saída da coluna, melhorou o sinal de UV. Determinou-se com este procedimento $\mathrm{Cu}, \mathrm{Zn}, \mathrm{Ni}, \mathrm{Mn}, \mathrm{Pb}$ e Co (todos bivalentes), com limite de detecção de $1 \mu \mathrm{g} \mathrm{g}^{-1}$, com regiões lineares de calibração variando de acordo com o metal em estudo.

$\mathrm{Li}$ et $\mathrm{al}^{6}$ apresentaram procedimento no qual $\mathrm{Ni}^{2+}$ e $\mathrm{Co}^{2+}$, foram determinados por CLAE, em fase reversa, na forma de quelatos de [2-(2-tiazoilazo)-p-cresol] (TAC). A coluna utilizada foi do tipo KYWG-C18 e a fase móvel consistiu de um tampão fosfato $(\mathrm{pH}=6,65)$ e TAC dissolvidos em metanol-THFágua (2:5:25). A detecção foi espectrofotométrica em $580 \mathrm{~nm}$. Os limites de detecção descritos foram $0,2 \mathrm{ng} \mathrm{mL}^{-1}$ para o $\mathrm{Ni}$ e $1,5 \mathrm{ng} \mathrm{L}^{-1}$ para o Co.

Miura e Tokunaga ${ }^{7}$, apresentaram um estudo bastante semelhante ao anterior, usando como fase móvel solução de acetonitrila aquosa (46\%), contendo brometo de tetrabutilamônio, acetato de sódio e EDTA. A coluna empregada foi do tipo Cosmosil 5 TMS $(25 \mathrm{~cm} \times 4,6 \mathrm{~mm})$, usando-se detector espectrofotométrico em $595 \mathrm{~nm}$. Os limites de detecção foram 2,2, 0,72, 16,5 e 98,8 ppb de V, Co, Ni, e Fe respectivamente.

$\mathrm{Li}$, Fan e Jiang ${ }^{8}$ mostraram um estudo na mesma linha dos anteriores, com coluna YWG-C18 e fase móvel constituindo-se de uma solução de brometo hexadeciltrimetilamônio, EDTA, e tampão fosfato em meio de metanol de $70 \%$. A detecção foi feita em $520 \mathrm{~nm}$, determinando-se concentrações de $\mathrm{Co}(\mathrm{II})$ e $\mathrm{Ni}$ (II) na faixa de 0,04 a $5,6 \mu \mathrm{g} \mathrm{mL}^{-1}$ com limites de detecção de 8-10 ppb, respectivamente e coeficiente de variação entre 0,7 a $1,2 \%$.

Foi descrito por Wang e $\mathrm{Liu}^{9}$ um procedimento para a determinação de $\mathrm{Cd}(\mathrm{II}), \mathrm{Pb}$ (II), $\mathrm{Co}(\mathrm{II}), \mathrm{Ni}(\mathrm{II}), \mathrm{Cu}(\mathrm{II})$ e $\mathrm{Hg}(\mathrm{II})$, usando como complexante o bis-2-hidroxietil-ditiocarbamato (cuja a síntese é descrita). A separação dos complexos foi efetuada utilizando-se uma coluna de Nucleosil C18 e uma fase móvel contendo um tampão fosfato $\mathrm{pH}=6$ e hidróxido de tetrabutilamônio, dissolvidos em metanol $40 \%$. A diferença em relação aos artigos anteriores citados é o tipo de detecção, que neste caso foi eletroquímico a $0,9 \mathrm{~V}$ contra ECS. Mostraram-se interferentes As e Se. As curvas analíticas foram lineares nas faixas de 0,005-1 (Co e Ni), 0,032-2,5 (Cu) e 0,5-10 $\mathrm{g} \mathrm{mL}^{-1}(\mathrm{Hg})$, com limites de detecção de $0,1,0,6$, e $10 \mathrm{ng} \mathrm{mL}^{-1}$ respectivamente.

A determinação simultânea de $\mathrm{Co}(\mathrm{II}), \mathrm{Cu}(\mathrm{II}), \mathrm{Fe}(\mathrm{II}), \mathrm{Fe}(\mathrm{III})$, $\mathrm{Zn}$ (II) $\mathrm{Pb}$ (II), Ni(II) foi discutida por Ou, Fu e Huang ${ }^{10}$. A coluna de cromatografia iônica utilizada foi a CS2, a fase móvel foi uma solução aquosa de ácido oxálico, ácido cítrico, $\mathrm{HCl}$ e ácido acético, em $\mathrm{pH}$ ajustado em 4 . O eluente contendo os íons reagiu com uma mistura de PAR, ácido acético, e amônia. A detecção foi feita em $520 \mathrm{~nm}$. In(IV), Bi(III), Mn(II), Cd(II), $\mathrm{Cr}$ (III) e $\mathrm{Hg}$ (II) não apresentaram interferência. As curvas analíticas foram lineares entre $0,025-0,5 / \mathrm{Fe}(\mathrm{III}), 0,1-1 / \mathrm{Cu}(\mathrm{II})$,
0,025-2,5/Ni(II), 0,1-5/Zn(II), 0,025-5/Co(II) e 0,4-1 ppm/Pb(II), com limites de detecção da ordem de $\mathrm{ng} \mathrm{L}^{-1}$, dependendo do metal e coeficientes de variação em torno de $2 \%$.

Xie, Huang e $\mathrm{Fu}^{11}$ analisaram $\mathrm{Cu}, \mathrm{Fe}, \mathrm{Ni}, \mathrm{Sn}, \mathrm{Co}$ e $\mathrm{Pb}$ em amostras de cabelo, por cromatografia iônica. A coluna utilizada foi HPIC-CS2, com fase móvel composta por ácido nítrico, ácido oxálico, ácido acético, nitrato de lítio e HCl. Após passarem pela coluna os metais reagiram com PAR, formando-se compostos coloridos, detectados em $520 \mathrm{~nm}$. A altura dos picos foi então relacionada com a concentração. Os coeficientes de recuperação foram da ordem de $85-106 \%$.

Chen e $\mathrm{Guo}^{12}$ descreveram a aplicação de uma coluna do tipo HPIC-CS2, modificada, utilizando como fase móvel ácido oxálico e ácido cítrico, quando na ausência de Mn e, ácidos tartárico e cítrico, na presença daquele cátion. O reagente cromóforo foi o PAR. Desta forma os autores determinaram simultaneamente $\mathrm{Cu}(\mathrm{II}), \mathrm{Zn}, \mathrm{Co}(\mathrm{II}), \mathrm{Mn}(\mathrm{II}), \mathrm{Ni}(\mathrm{II})$, com recuperações entre $96-104 \%$.

Utilizando complexos de PAR com $\mathrm{Fe}$, Ni e Co preparados previamente, Ji, Cao e Wang ${ }^{13}$ fizeram estudos de determinações destes metais usando uma coluna YWG-CD ODS, usando como fase móvel tampão fosfato $(\mathrm{pH}=7)$-metanol, 1:1. A detecção eletroquímica foi feita a $1,2 \mathrm{~V}$ (em relação ao ECS). Não são apresentados detalhes sobre os resultados.

A separação cromatográfica de metais pesados na forma de seus complexos com ditiocarbamatos, foi discutida por Irth et $\mathrm{al}^{14}$. Os complexos de $\mathrm{Pb}(\mathrm{II}), \mathrm{Hg}(\mathrm{II}), \mathrm{Cu}(\mathrm{II}), \mathrm{Co}(\mathrm{II}), \mathrm{Ni}$ e $\mathrm{Bi}$, com dietilditiocarbamato foram formados por reação em pré coluna (2 $\mathrm{mm}$ x 4,6 mm), preenchida com Spherisorb ODS ou Hypersil OSD e carregada com solução $\mathrm{pH}=5,5$ de brometo de hexadeciltrimetilamônio e dietilditiocarbamato. As separações foram efetuadas utilizando-se colunas Spherisorb ODS ou Hypersil OSD, eluindo-se a mistura com tampão fosfato $(\mathrm{pH}=6,8)$ e acetonitrila (3:2 a 1:3). A detecção espectrofotométrica foi feita em $254 \mathrm{~nm}$.

Roston ${ }^{15}$ descreveu um trabalho de determinação simultânea de $\mathrm{Fe}(\mathrm{II}), \mathrm{Co}(\mathrm{II}), \mathrm{Ni}(\mathrm{II})$ e $\mathrm{Cu}(\mathrm{II})$, no qual os metais são previamente tratados com PAR e os complexos formados então separados em coluna Biofase-OSD. A fase móvel utilizada foi uma mistura de tampão fosfato de amônio $\mathrm{pH}=6,5$ e metanol (7:13), com detecção espectrofotométrica $(254 \mathrm{~nm})$ ou amperométrica $(+1,1 \mathrm{~V})$. Usando métodos de adição de padrão o autor afirma ser possível determinar os metais ao nível de $\mathrm{ppb}$.

Bond e Wallace ${ }^{16,17,18}$ relataram a determinação simultânea de $\mathrm{Co}, \mathrm{Cu}, \mathrm{Cd}, \mathrm{Pb}, \mathrm{Hg}$, Ni e $\mathrm{Zn}$, na forma de sulfatos, utilizando em cada trabalho um ditiocarbamato diferente, propondo gerálos "in situ". Em todos os casos, a separação dos complexos foi efetuada por CLAE, utilizando coluna do tipo C18, com diferentes composições para a fase móvel. A detecção foi espectrofotométrica ou eletroquímica. A influência da utilização de cada detector nos resultados foi discutida, além de testes com diferentes tipos de eletrodo, como $\mathrm{Au}, \mathrm{Pt}$ e carbono vítreo.

Utilizando-se de uma coluna Cosmosil 5 C18, Ichinoki, Morita e Yamasaki ${ }^{19}$ propuseram um procedimento para a determinação simultânea de $\mathrm{Cd}$, Ni(II), $\mathrm{Co}(\mathrm{II}), \mathrm{Cu}(\mathrm{II}), \mathrm{Bi}(\mathrm{III})$, e $\mathrm{Hg}(\mathrm{II})$. Os metais foram complexados com hexametilditiocarbamato, em clorofórmio em $10^{\circ} \mathrm{C}$. A fase móvel utilizada foi metanol, água, éter etílico, tampão de amônia e o hexametilditiocarbamato (82:9:3:3:3), com uma detecção espectrofotométrica em $260 \mathrm{~nm}$. Como resultados foram apresentados os níveis de interferência para 22 espécies, os coeficientes de recuperação, que foram de 99,2-101,5\%, com gráficos lineares em nível de ppb. Para os íons metálicos, na ordem acima descrita, os limites de detecção foram $45,150,75,150,150$ e $300 \mathrm{ppb}$, respectivamente.

\section{ELETROANALÍTICA}

Complexos de Co e Ni com dimetilglioxima, geralmente utilizados para determinação simultânea dos metais, foram investigados por Vulkomanovic, Page e van Loon ${ }^{20}$. Estes complexos 
são normalmente utilizados em voltametria de redissolução adsortiva e, neste caso, os autores utilizaram diversas técnicas para explicar a elevada sensibilidade do método. Foram obtidos polarogramas dc entre $-0,4$ e $-1,8 \mathrm{~V}$, medidas de corrente em função do tempo utilizando gotas pendentes de mercúrio, com área de $1,18 \mathrm{~mm}^{2}$, com intervalos de tempo de $4 \mathrm{~s}$ e, finalmente, medidas de redução de complexos adsorvidos a $-0,85 \mathrm{~V}$, com tempo de pré-concentração da ordem de $2-14$ s, sendo realizadas varreduras de dessorção para potenciais mais negativos. Os resultados apontaram para processos de redução envolvendo 16 ou 18 elétrons.

Um método para análise simultânea de Co e Ni, em nível de traço, por voltametria de redissolução catódica, foi descrito por Zhou et $\mathrm{al}^{21}$. Os autores utilizaram os reagentes benzoína e tiosemicarbazida, em meio de tampão amoniacal, $\mathrm{pH}=8,6$. O tempo de pré-concentração foi de $60 \mathrm{~s}$ a $-0,3 \mathrm{~V}$. Os processos de redução resultaram em picos em $-0,94$ e $-0,75 \mathrm{~V}$, contra o ECS, para Co e Ni, respectivamente. As curvas analíticas foram lineares entre 20-1000 (Co) e 10-80 $\mathrm{nmol} \mathrm{L}^{-1}(\mathrm{Ni})$, com recuperações de 98-101,8 e 97,9-102,1\% e RSD 1,4-2 e 2,12,5\% respectivamente para cada espécie.

Paneli e Voulgaropulos ${ }^{22}$ desenvolveram estudos sobre a determinação simultânea de $\mathrm{Co}, \mathrm{Ni}$ e $\mathrm{Zn}$ usando reagente 2quinolinatiol e triton X-100 (surfactante) em águas de estuários. Para tanto o pH da amostra de água foi ajustado em 9,2 com hidróxido de amônio para posterior agitação com solução etanólica do reagente, em concentração de $0,01 \mathrm{mmolL}^{-1}$. O níquel e o cobalto foram determinados por análise de redissolução adsortiva utilizando voltametria de pulso diferencial. Os eletrodos utilizados foram eletrodos de gota pendente de mercúrio (EGPM-trabalho), $\mathrm{Ag} / \mathrm{AgCl}$ (referência) e fio de platina (auxiliar). O tempo de adsorção foi de 1 minuto, com velocidade de varredura de 10 $\mathrm{mVs}^{-1}$, na fase dessortiva. O uso de Triton X-100 não apresentou interferência, o que tornou desnecessária a digestão ácida ou irradiação por ultravioleta. A presença do surfactante resultou em uma melhor separação dos picos e Ni e Co. Os limites de detecção descritos são da ordem de $1 \mathrm{mmol} \mathrm{L}^{-1}(\mathrm{Ni}), 10 \mathrm{mmol} \mathrm{L}^{-1}(\mathrm{Co})$ e $1 \mu \mathrm{mol} \mathrm{L}{ }^{-1}(\mathrm{Zn})$, com RSD de $6,3 \%$.

A determinação de $\mathrm{Zn}$, Co, Ni por polarografia de regressão ortogonal foi descrita por Wang, Zhao, e Jiao ${ }^{23}$. O procedimento adotado foi a utilização de três tubos denominados A, B, C. Nos três tubos foram adicionados solução teste contendo os três íons, etilenodiamina (EDA) e tartarato misto de sódio e potássio. No tubo B adicionou-se ainda sulfosalicilato de sódio (SSS). No tubo C adicionou-se nitrito de sódio e SSS. Das soluções resultantes traçaram-se polarogramas no intervalo de potencial entre $-1,1$ e $-1,6 \mathrm{~V}$ (em relação ao $\mathrm{Ag} / \mathrm{AgCl}$ ), com velocidade de varredura $1,50 \mathrm{mV} \mathrm{s}^{-1}$. Obtiveram-se correntes de pico em -1,38 V (A), -1,40 V (B), e -1,32 V (C), as quais foram relacionadas com as concentrações de $\mathrm{Zn}$, Co e $\mathrm{Ni}$ em amostras de solo e vegetais. Obtiveram-se coeficientes de recuperação da ordem de $95-108 \%$ com RSD $(n=5)$ de 5,3\%.

Liang e $\mathrm{She}^{24}$ demostraram o aproveitamento de ondas catalíticas polarográficas de $\mathrm{Co}$ e $\mathrm{Ni}$, em presença de dimetilglioxima, nitrito de sódio, ácido sulfasilisílico e cloreto de amônio, -1,27 e -1,08 V em relação ao ECS. Usando-se estas ondas, os autores conseguiram determinar $\mathrm{Ni}$ e $\mathrm{Co}$ na faixa de $1 \mathrm{ng}-1 \mu \mathrm{g} \mathrm{mL} \mathrm{m}^{-1}$ simultaneamente. Segundo os autores o método foi aplicado na análise de óxido de cobre de alta pureza e minerais de cobalto com alta e boa seletividade.

Uma proposta para resolver numericamente problemas associados à superposição de ondas polarográficas na determinação simultânea de $\mathrm{Cd}, \mathrm{Ni}, \mathrm{Zn}, \mathrm{Co}, \mathrm{Fe}$, e $\mathrm{Mn}$ foi apresentada por She e $\mathrm{Wang}^{25}$. Polarogramas de soluções contendo metais e às quais se adicionaram tampões de amônia e soluções $\mathrm{KCl}$, sulfito e salicilato foram obtidos e as ondas sobrepostas puderam ser resolvidas utilizando-se um programa de computador escrito em linguagem BASIC, baseado na equação de filtragem de Kalman. As curvas analíticas foram lineares na faixa de 0,01-
$140 \mu \mathrm{g} \mathrm{mL} \mathrm{m}^{-1}$, dependendo do cátion. Os coeficientes de recuperação foram da ordem de 83,5-106\%.

A aplicação da oscilopolarografia para a determinação simultânea de $\mathrm{Ni}$ e Co em Zinco eletrolítico foi estudada por $\mathrm{Chen}^{26}$. Os reagentes utilizados na complexação foram o clorihidrato de hidroxilamina, fluoreto de amônio e citrato de amônio. Após a complexação a mistura foi basificada com tampão amônia até a viragem da fenolftaleína e os polarogramas traçados na presença de nitrito de sódio. Picos entre -0,98 e $1,03 \mathrm{~V}$ e $-1,16 \mathrm{e}-1,22 \mathrm{~V}$ em relação ao ECS forneceram informações sobre as concentrações de $\mathrm{Ni}$ e $\mathrm{Co}$, respectivamente. Curvas analíticas lineares foram obtidas para $0,4 \mathrm{ng} \mathrm{mL}^{-1}$ $0,2 \mu \mathrm{g} \mathrm{mL}^{-1}$ de $\mathrm{Ni}^{2+}$ e $0,2-2 \mathrm{ng} \mathrm{mL}^{-1}$ de $\mathrm{Co}^{2+}$.

A utilização da técnica de deconvolução de terceira derivada para a determinação simultânea de $\mathrm{Ni}$ e Co em bebidas, usando dimetilglioxima em pH 9,2 e ondas adsortivas, foi descrita no trabalho de Yao, Chen e Fang ${ }^{27}$. Após a pré-concentração de 30 segundos em $-0,75 \mathrm{~V}$ (em relação ao ECS), traçaram-se os polarogramas com velocidade de varredura de $60 \mathrm{mVs}^{-1}$, usando-se um EGPM, como eletrodo de trabalho. Os coeficientes de recuperação foram da ordem de 95-104\%, com limites de detecção de 0,03 e $0,05 \mu \mathrm{gL}^{-1}$ para $\mathrm{Ni}$ e Co, respectivamente. Foi observada a interferência de surfactantes.

Sharma, Kumbaht e Rawat ${ }^{28}$, determinaram traços de Ni e Co em águas naturais por polarografia de pulso diferencial, utilizando como reagente a furilidoxima. A amplitude de modulação foi de $50 \mathrm{mV}$ e velocidade de varredura de $5 \mathrm{mV} \mathrm{s}^{-1}$ com EGPM. Foram obtidos picos em -0,86 e -1,04 V (ECS), para $\mathrm{Ni}$ e $\mathrm{Co}$, respectivamente. Relatam-se os limites de detecção da ordem de 0,4 e $0,15 \mu \mathrm{g} \mathrm{mL}^{-1}$ e coeficientes de variação de 7 e $8,3 \%$ para $\mathrm{Ni}^{2+}$ e $\mathrm{Co}^{2+}$, respectivamente.

A determinação de $\mathrm{Cu}, \mathrm{Ni}, \mathrm{Pb}, \mathrm{Co}$ e $\mathrm{Cd}$ (bivalentes), foi discutida por Zhang, Chen e Zhang ${ }^{29}$, utilizando polarografia convencional, de pulso diferencial e voltametria cíclica. Ondas dessortivas dos metais com 1-(2-piridilazo)-2,7-dihidroxinaftaleno (cuja preparação é descrita), foram detectadas em -0,35, $0,45,-0,49,-0,60$ e $-0,67 \mathrm{~V}$, respectivamente. As curvas analíticas foram lineares na ordem de $\mathrm{ng} \mathrm{ml}^{-1}$, variando de acordo com o metal.

Economou e Fieldem ${ }^{30}$ realizaram estudos de redissolução adsortiva com voltametria de onda quadrada para a determinação simultânea de $\mathrm{Co}(\mathrm{II})$ e $\mathrm{Ni}(\mathrm{II})$, utilizando eletrodo rotativo com filme de mercúrio. Os metais foram complexados com dimetilglioxima em meio amoniacal $(\mathrm{pH}=9)$ e pré-concentrados a $-0,7 \mathrm{~V}$ $(\mathrm{Ag} / \mathrm{AgCl})$, com eletrodo rotatório $(10 \mathrm{~Hz})$, por 120 segundos. Varredura de potencial até $-1,3 \mathrm{~V}$ levou a ondas bem definidas dos dois complexos, o que permitiu a determinação simultânea dos íons metálicos em condições em que uma das espécies estava em concentração 100 vezes maior que a do outro. Observou-se que o zinco interfere em excesso de mais de 500 vezes e surfactantes tendem a suprimir a corrente de pico. As curvas analíticas foram lineares acima de $12 \mu \mathrm{g} \mathrm{mL}{ }^{-1}$, com limites de detecção da ordem de $14 \mathrm{ng} \mathrm{L}^{-1}$. Coeficientes de variação $(n=8)$ de 2-3\% foram estimados para concentrações de $12 \mu \mathrm{g} \mathrm{mL}{ }^{-1}$.

Um procedimento polarográfico derivativo para a determinação de $\mathrm{Cu}, \mathrm{Ni}$, Co e $\mathrm{Cd}$, usando 1-(2-quinolilazo)-2,7-dihidroxinaftaleno, em meio amoniacal $(\mathrm{pH}=9,6)$. As ondas polarográficas foram observadas e, $-0,37,-0,69,-0,77$ e $-0,82 \mathrm{~V}$, respectivamente. A linearidade foi verificada entre $0,08-3 \mu \mathrm{g} \mathrm{mL}^{-1}$, com limites de detecção variando na faixa de $20-50 \mu \mathrm{g} \mathrm{mL}^{-1}$ e coeficientes de recuperação de $91-108 \%$. O trabalho foi proposto por Zhang et $\mathrm{al}^{31}$.

Paneli e Voulgaropoulus ${ }^{32}$, descreveram um método voltamétrico adsortivo de pulso diferencial usando o reagente 2quinolinatiol, para determinação simultânea de $\mathrm{Ni}$ (II) e $\mathrm{Co}(\mathrm{II})$, em um potencial de adsorção de $-0,8 \mathrm{~V}$ e tempo de pré- concentração de 2 minutos, com picos separados por $0,3 \mathrm{~V}$ com limites de detecção de 1 e $10 \mathrm{nmol} \mathrm{L} \mathrm{L}^{-1}$, respectivamente. $\mathrm{Pb}$ e $\mathrm{Cd}$, não interferiram. 
Um estudo para a determinação simultânea de $\mathrm{Cu}(\mathrm{II}), \mathrm{Pb}(\mathrm{II})$, $\mathrm{Zn}(\mathrm{II}), \mathrm{Fe}(\mathrm{II}), \mathrm{Co}(\mathrm{II}), \mathrm{Ni}(\mathrm{II})$ e $\mathrm{Mn}(\mathrm{II})$ em alimentos, foi proposto por Feng e Chen ${ }^{33}$. Após a abertura da amostra por tratamento nítrico-perclórico, adicionou-se ácido tartárico e determinou-se $\mathrm{Zn}, \mathrm{Cd}, \mathrm{Pb}$, e $\mathrm{Cu}$, por voltametria anódica de onda quadrada entre $1,2-0,15 \mathrm{~V}$ (em relação ao $\mathrm{Ag} / \mathrm{AgCl}$ ). O Fe e o $\mathrm{Mn}$, foram determinados após o ajuste de $\mathrm{pH}$ em 9,5, com amônia entre 1,2 a $-1,7$, pela mesma técnica. Finalmente, após a adição de DMG, determinou-se o Ni e o Co entre -0,9 e -1,3V. Coeficientes de variação entre $0,95-13,3 \%$ foram descritos.

Locatelli, Fagioli e Garai ${ }^{34}$ estudaram a determinação simultânea de $\mathrm{Co}(\mathrm{II})$ e Ni(II), por polarografia de pulso diferencial e por polarografia ac de primeira e segunda harmônicas. $\mathrm{O}$ eletrodo gotejante utilizado foi ajustado para tempos de vida de gota de 3 a 4 minutos. O ECS foi utilizado como referência e o fio de platina como auxiliar. O meio analítico consistiu de um tampão de amônia e o complexante a DMG. Quanto à seletividade, o melhor desempenho foi verificado com polarografia ac de $2^{a}$ harmônica, seguida de pulso diferencial e finalmente ac-fundamental. Os melhores limites de detecção descritos foram 0,66 e 0,74 nmol L ${ }^{-1}$, respectivamente para Co e $\mathrm{Ni}$.

Em meio amoniacal $(\mathrm{pH}=9)$, complexos de $\mathrm{Co}(\mathrm{II})$ e $\mathrm{Ni}(\mathrm{II})$ com nitroso-R, apresentam ondas de redução bem definidas no EGM, em -0,57V ( em relação ao ECS). Utilizando esta informação, $\operatorname{Tan}^{35}$ propôs um método para a determinação simultânea de $\mathrm{Co}$ e $\mathrm{Ni}$, que foi aplicado a soluções contendo estes cátions em concentrações acima de 0,4 e $0,8 \mu \mathrm{g} \mathrm{mL}^{-1}$, respectivamente. $\mathrm{O}$ erro relativo em 9 soluções testadas foi de $8 \%$, tendo-se analisado o limite de interferência para 22 íons.

Liang e Shi ${ }^{36}$ aproveitaram o fato de que tanto o $\mathrm{Ni}$ e o Co apresentam ondas polarográficas catalíticas, em meio amoniacal e em presença de DMG, nitrito de sódio e ácido sulfossalicílico, para sua determinação simultânea. Estas ondas surgem em -1,08 e $-1,27 \mathrm{~V}$ em relação ao ECS, respectivamente. As curvas ana-

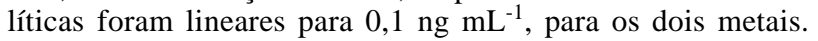

A utilização da técnica adsortiva de redissolução com varredura catódica em voltametria de onda quadrada, para a determinação simultânea de $\mathrm{Co}$ e $\mathrm{Ni}$ em água do mar foi descrita por Zhang et $\mathrm{al}^{37}$. O pH das amostras foi controlado, com tampão amônia/trietanolamina $(\mathrm{pH}=8,0)$, o complexante utilizado foi DMG. As condições eletroquímicas foram: pré-concentração a 0,7 V (em relação ao $\mathrm{Ag} / \mathrm{AgCl}$ ), por 1-3 minutos, a etapa de redissolução foi feita a $70 \mathrm{~Hz}$, com pulsos de $25 \mathrm{mV}$ e passo de $2 \mathrm{mV}$. Os picos se localizaram em $-0,98$ e $-1,12 \mathrm{~V}$, com limites de detecção de 50 e $8 \mu \mathrm{g} \mathrm{mL}^{-1}$ respectivamente para o Ni e Co.

A determinação simultânea de $\mathrm{Ni}$ e $\mathrm{Co}$, na forma de seus complexos ternários com 2,2'-(pentano-2,4-diildenodiamino)difenol em presença de piridina por polarografia de pulso diferencial, foi apresentada por Odashima, Kawate e Ishii ${ }^{38}$. Nestas condições o $\mathrm{Co}(\mathrm{II})$ é facilmente oxidado a $\mathrm{Co}$ (III), pelo oxigênio presente. Os complexos são extraídos em 4-metil-1,3-dioxalano-2-ona, em presença de perclorato de tetrabutilamônio, como eletrólito suporte. Os polarogramas apresentam ondas bem definidas em -0,44 e $1,20 \mathrm{~V}$ (em relação ao ECS), correspondendo às reduções do $\mathrm{Co}(\mathrm{III})$ e $\mathrm{Ni}(\mathrm{II})$, respectivamente.

O comportamento polarográfico e as potencialidades dos complexos de $\mathrm{Cd}(\mathrm{II}), \mathrm{Fe}(\mathrm{II}), \mathrm{Co}(\mathrm{II}), \mathrm{Ni}(\mathrm{II})$ e $\mathrm{Zn}(\mathrm{II})$ com piridina, na determinação destes metais foram estudados por Saraswathi ${ }^{39}$. Foram utilizados como agentes supressores de precipitação manitol para Fe e acetato de amônio para os demais cátions e nitrato de potássio como eletrólito suporte. Curvas analíticas lineares foram obtidas entre $0,5-2 \mathrm{mmol} \mathrm{L}^{-1}$. Testes em várias misturas binárias mostraram valores e limite de detecção e desvios característicos, para cada para íon metálico estudado.

A determinação direta de $\mathrm{Ti}(\mathrm{IV}), \mathrm{Cu}(\mathrm{II})$ e $\mathrm{Pb}(\mathrm{II})$, ou Ti(IV), $\mathrm{Ni}(\mathrm{II})$ e Co(II), foi discutida por Kurtov e Nikitina ${ }^{40}$. As amostras foram dissolvidas em mistura de ácido fosfórico/pirofosfórico, para evitar hidrólise do Ti. A polarografia convencional foi empregada para a determinação direta em amostras de primeiro tipo, sem a interferência de $\mathrm{Zn}, \mathrm{Ni}(\mathrm{II})$ ou $\mathrm{Co}(\mathrm{II})$, em concentrações 100 vezes maiores. Para amostras do segundo tipo, o Ti é analisado diretamente e o $\mathrm{Co}$ e $\mathrm{Ni}$, determinados após a adição de amônia em pH=9-11, sem que se tenha constatado interferência de $\mathrm{Fe}, \mathrm{Cr}(\mathrm{III}), \mathrm{Si}, \mathrm{F}^{-}, \mathrm{Cu}(\mathrm{II})$ ou $\mathrm{Zn}(\mathrm{II})$, em concentrações 100 vezes maiores, que a dos analitos.

Estudos de determinação simultânea de Co e Ni em sulfato de manganês, foram desenvolvidos por Adeloju e Tran ${ }^{41}$. As amostras foram tamponadas com acetato de amônio e tratadas com DMG, sendo $\mathrm{pH}=7,7$ o ideal para trabalho. Os eletrodos utilizados foram EGPM em relação a $\mathrm{Ag} / \mathrm{AgCl}$. A adição de padrões dos metais a analisar, apresentaram picos em - 0,9 e $-1,0 \mathrm{~V}$, respectivamente. Os limites de detecção foram de 2 e $1 \mu \mathrm{g} \mathrm{mL} \mathrm{mL}^{-1}$, com coeficientes de variação de $1-3 \%$. As curvas analíticas se mostraram lineares acima de 110 e $140 \mu \mathrm{g} \mathrm{mL}^{-1}$ para Ni e Co respectivamente.

A determinação simultânea de traços de $\mathrm{Cu}(\mathrm{II}), \mathrm{Pb}(\mathrm{II})$, $\mathrm{Cd}(\mathrm{II}), \mathrm{Zn}(\mathrm{II}), \mathrm{Ni}(\mathrm{II})$ e $\mathrm{Co}(\mathrm{II})$ em impressões digitais, foi descrita por Jin e $\mathrm{Xu}^{42}$, usando voltametria de pulso diferencial. Para isso na mesma solução, determinaram-se $\mathrm{Cu}, \mathrm{Pb}$ e $\mathrm{Cd}$ por redissolução anódica, com EGPM, observando-se picos em $+0,021,-0,385$ e $-0,582 \mathrm{~V}$, em meio perclorato. $\mathrm{O} \mathrm{Zn}$ foi determinado por voltametria catódica em $-1,175 \mathrm{~V}$ em meio amoniacal e finalmente o Co e $\mathrm{Ni}$ em $-1,128$ e $-0,974 \mathrm{~V}$, na forma de seus complexos com DMG, por adsorção e voltametria de pulso diferencial.

Jin et $\mathrm{al}^{43}$ aplicaram a voltametria adsortiva em EGPM, para a determinação simultânea de Ni e Co, baseando-se na adsorção de complexos de DMG, na superfície do eletrodo e dessorção por varredura linear do potencial, em um processo de redissolução.

A polarografia ac, foi usada por Sviridenko et $\mathrm{al}^{44}$, para a determinação simultânea de traços de $\mathrm{Fe}, \mathrm{Ni}$ e Co em materiais poliméricos. Após a queima do material orgânico e dissolução ácida dos metais, em presença de oxalato de amônio traçaramse polarogramas de onda quadrada em eletrodo de poça de $\mathrm{Hg}$. Foi observada uma onda de redução do Fe(III) em -0,85V, sem interferência de Co em concentrações até 500 e de Ni até 700 vezes maior. Tratamento posterior com ácido cítrico e ajuste de $\mathrm{pH}$ em 8,5 com amônia, forneceu picos ac em -0,96(Ni) e $1,38 \mathrm{~V}(\mathrm{Co})$. O coeficiente de variação $(\mathrm{n}=6)$ foi de $12 \%$, determinando-se 2-24 (Co), 2-20 (Ni) e 1-12 ppm (Fe).

Em 1984 Markova e Shirokova ${ }^{45}$ descreveram metodologia para a determinação de $\mathrm{Ni}$ e $\mathrm{Co}$, usando microeletrodo de $\mathrm{Hg}$. Os cátions foram pré-concentrados no EGPM, mantido a $-1,2 \mathrm{~V}$ (em relação ao ECS), em $\mathrm{pH}=6$, por 5 minutos. Os picos de oxidação foram detectados em $-0,20$ e $-0,24 \mathrm{~V}$. As alturas dos picos relacionaram-se linearmente com as concentrações de $\mathrm{Ni}$ (II) entre 0,1 e 100 e de $\mathrm{Co}$ (II) $0,3-10 \mu \mathrm{g} \mathrm{mL} \mathrm{m}^{-1}$. A determinação simultânea só foi possível dopando-se o eletrodo com $\mathrm{Au}(\mathrm{III})$ a $-0,9 \mathrm{~V}$ por 30 minutos. Com o eletrodo dopado foi possível determinar 5-20ng $\mathrm{mL}^{-1}$ de Ni e $10-30 \mathrm{ng} \mathrm{mL}^{-1}$ de Co, em amostras preparadas.

A determinação simultânea de $\mathrm{Co}(\mathrm{II})$ e $\mathrm{Ni}(\mathrm{II})$ em amostras biológicas foi estudada por Adeloju, Bond e Briggs ${ }^{46}$, utilizando-se de voltametria de pulso diferencial em processo de redissolução, de complexos dos metais com DMG em meio amoniacal. A deposição foi efetuada em um EGPM, com tempos entre 15 e 225 segundos a $-0,8 \mathrm{~V}$ ( em relação ao eletrodo $\mathrm{Ag} / \mathrm{AgCl}$ ). A varredura de redissolução foi feita entre $-0,6 \mathrm{e}$ $1,4 \mathrm{~V}$, com picos a $-1,15$ e $1,03 \mathrm{~V}$, identificados por adição de padrão como sendo respectivamente dos complexos de $\mathrm{Ni}$ e Co. Limites de detecção foram 0,01 (Co) e $0,02 \mu \mathrm{g} \mathrm{g}^{-1}$ (Ni), em rins bovinos.

Ostapczuk et $\mathrm{al}^{47}$ descreveram a determinação de $\mathrm{Zn}, \mathrm{Cd}, \mathrm{Pb}$, $\mathrm{Cu}$, Ni e Co, por voltametria anódica de redissolução-pulso diferencial. Neste procedimento os quatro primeiros cátions foram determinados diretamente no eletrólito de suporte (ácido perclórico), em EGPM. O Ni e Co foram determinados na forma 
de seus DMG-complexos. Limites de detecção da ordem de $1 \mathrm{ng} \mathrm{\textrm {L } ^ { - 1 }}$, foram obtidos.

Complexos de xantatos derivados de compostos com cadeias lineares e ramificadas de 3-5 átomos de carbono, foram utilizados na determinação simultânea de $\mathrm{Fe}(\mathrm{II}), \mathrm{Ni}(\mathrm{II})$ e $\mathrm{Co}(\mathrm{II})$, num método apresentado por Budnikov, Ulakhovich e Postnova $^{48}$. A oxidação dos complexos formados em eletrodos de platina ou carbono vítreo, permitiu a determinação dos metais com limites de detecção da ordem de $0,3 \mu \mathrm{g} \mathrm{mL}{ }^{-1}$. A aplicação de voltametria ac, melhorou a detecção de Fe.

Titulações amperométricas para análise de misturas contendo $\mathrm{Bi}-\mathrm{Cd}, \mathrm{Cu}-\mathrm{Pb}$ e $\mathrm{Co}-\mathrm{Ni}$, foram efetuadas com morfolinoditiocarbamato de sódio, por Rao et $\mathrm{al}^{49}$. $\mathrm{O} \mathrm{pH}$ das soluções foi ajustado para $6,8,8,5$ e 8,5 respectivamente e o primeiro metal determinado num EGPM em relação ao ECS a -0,2V (Cd ou $\mathrm{Co})$ e $-0,10 \mathrm{~V}(\mathrm{~Pb})$, após a precipitação do primeiro cátion analisou-se o segundo, de maneira semelhante.

\section{ESPECTROFOTOMETRIA}

Li e $\mathrm{Xu}^{50}$ realizaram estudos para determinação simultânea de $\mathrm{Fe}, \mathrm{Co}$ e $\mathrm{Ni}$, por espectrofotometria, utilizando o método quemométrico. A determinação das absorbâncias em 520, 550 e $720 \mathrm{~nm}$, foram feitas após tratamento das amostras com clorohidrato de hidroxilamina e p-nitrofenol, em $\mathrm{pH}=5,5$ ajustado com tampão acetato. A Lei de Beer foi seguida até $3(\mathrm{Fe})$, $3(\mathrm{Ni})$ e $4 \mu \mathrm{g} \mathrm{mL} \mathrm{m}^{-1}(\mathrm{Co})$. Foi observada interferência de $\mathrm{Cu}$, sendo o método aplicado em ligas de $\mathrm{Zn}$, com coeficiente de recuperação entre $95-110 \%$, e RSD de $1,7-7,8 \%$.

Utilizando espectrofotometria de multi-comprimentos de onda, Garcia Rodriguez et $\mathrm{al}^{51}$, apresentaram um procedimento para determinação de $\mathrm{Co}, \mathrm{Ni}$ e $\mathrm{Fe}$, utilizando um meio de ácido ascórbico, 1,5-bis(di-2-piridilmetileno) tiocarbonohidrazina (DPTH) e DMF, com tampão acetato em pH=4. A absorbânica da solução resultante foi medida, após 30 minutos, entre 390-510 nm, contra DPTH. Um programa de computador chamado de MULTIC foi utilizado na determinação das concentrações. As curvas analíticas foram lineares entre $0,2-1,3(\mathrm{Co})$, $0,1-1,2(\mathrm{Ni})$ e $0,1-1,1 \mu \mathrm{g} \mathrm{mL} \mathrm{m}^{-1}(\mathrm{Fe})$, com limites de detecção de $0,05\left(\mathrm{Fe}\right.$ e Ni) e $0,1 \mu \mathrm{g} \mathrm{mL} \mathrm{m}^{-1}(\mathrm{Co})$. Limites de tolerância para vários interferentes foram descritos, juntamente com resultados de aplicação a ligas, amostras sintéticas e amostras certificadas de tecidos biológicos.

Ren e $\mathrm{Gao}^{52}$ descreveram um procedimento de determinação para $\mathrm{Co}$, $\mathrm{Ni}$ e $\mathrm{Cu}$, simultaneamente utilizando EDTA, em $\mathrm{pH}=7$ 8. As absorbâncias de soluções contendo diferentes relações de concentração dos metais, foram determinadas em 379, 463, 585, 732 e 869nm, utilizando um método de regressão linear multivariada, sendo os cálculos efetuados com um programa chamado de SPGRMLR, cujos detalhes de uso são descritos no texto. Para estudos de espectro inteiro, as absorbâncias foram determinadas entre $370-875 \mathrm{~nm}$, com intervalos de 2 ou $5 \mathrm{~nm}$, após o que uma matriz de absorção foi montada e os dados tratados por transformadas de Fourrier, através de um outro programa computacional chamado de SPGRFSQ. Os autores concluíram que o segundo procedimento levou a melhores resultados.

Um procedimento direto para a determinação de $\mathrm{Ag}, \mathrm{Cd}$, $\mathrm{Pb}, \mathrm{Bi}, \mathrm{Cr}, \mathrm{Mn}, \mathrm{Co}, \mathrm{Ni}, \mathrm{Li}, \mathrm{Be}, \mathrm{Cu}, \mathrm{Sb}$, em amostras de água e materiais geológicos foi proposto por Sengupta e Bouvier ${ }^{53}$. Os autores utilizaram EAA, com forno de grafite e correção de efeito Zeeman. As amostras foram abertas por digestão com $\mathrm{HF} / \mathrm{HNO}_{3} / \mathrm{HCl}$, em forno de microondas, sendo posteriormente analisadas por EAA-FG, em presença de ácido bórico e EDTA. Os elementos foram determinados em diferentes grupos, com diferentes temperaturas de atomização. Os resultados são discutidos e curvas analíticas, apresentadas para cada elemento.

Estudos de espectroscopia de fluorescência de raios-X, para determinação de pequenas quantidades de $\mathrm{Fe}, \mathrm{Co}, \mathrm{Ni}, \mathrm{Cu}, \mathrm{Hg}$, $\mathrm{Pb}$, todos no estado bivalente, após a pré-concentração na forma de complexos de piperizino-1,4-bis(ditiocarbamato) de sódio, foram descritos por $\mathrm{Lau}$ e $\mathrm{Ho}^{54}$. Os precipitados formados foram pesados e secos. Os espectros foram obtidos em porta-amostras de plástico e utilizando-se um tubo de Rh de baixa potência. Fe, $\mathrm{Co}, \mathrm{Cu}$ e $\mathrm{Zn}$ foram determinados pelas linhas $\mathrm{K}$-alfa e enquanto $\mathrm{Hg}$ e $\mathrm{Pb}$, pelas linhas L-alfa. Os autores afirmam que as curvas analíticas são lineares (não apresentadas), com limites de detecção de $0,07-0,14 \mu \mathrm{g} \mathrm{mL}{ }^{-1}$ dependendo do metal. A percentagem de recuperação de soluções padrão multi-elementares foram de $97-105 \%$. O método foi aplicado em folhas de citros, ligas metálicas certificadas e água de mar e de rejeito.

Chen et $\mathrm{a}^{55}$ mediram a absorbância de soluções contendo $\mathrm{Cu}, \mathrm{Zn}, \mathrm{Co}, \mathrm{Ni}$ e $\mathrm{Mn}$, em dezenove comprimentos de ondas entre 530-580 nm. Soluções padrão foram tratadas com éter octilfenilpoliglicólico, 5-bromo-2-piridilazo-5-dietilaminofenol e tampão amônia / cloreto de amônio $(\mathrm{pH}=9)$. As curvas de calibração foram lineares acima de 1,52 (Cu), 1,28 ( $\mathrm{Zn}, \mathrm{Co}$ e Ni) e $0,96 \mu \mathrm{g} \mathrm{mL}^{-1}(\mathrm{Mn})$, com limite de detecção de 3,8-5,2 $\mathrm{ng} \mathrm{L}^{-1}$ e erro relativo de $10 \%$. Segundo os autores, o método foi testado em amostras geológicas e resultados de aplicação a seis amostras, foram consistentes com os valores certificados.

Rigin $^{56}$, construiu um equipamento para a determinação simultânea de $\mathrm{Fe}$, Co e Ni, usando espectrometria de fluorescência atômica, utilizando os metais nas formas de carbonilas e fase gasosa atomizada por plasma induzido por microondas. Amostras de águas naturais foram tratadas com tiouréia e trishidroximetil-aminometano e colocados em um "carbonilador". Neste equipamento passou-se um fluxo de $\mathrm{CO}_{2}$ gerado pela a decomposição de $\mathrm{CaCO}_{3}$ em presença de pó de $\mathrm{Zn}$. O gás de saída do "carbonilador", foi conduzido a um recipiente resfriado a $-60^{\circ} \mathrm{C}$. Após a reação, foi aplicado na entrada do recipiente, um fluxo de $120 \mathrm{~mL} \mathrm{~min}^{-1}$ de $\operatorname{Ar}-\mathrm{CO}(5: 1)$ e a sua saída foi conectada a um atomizador capilar de zircônia, montado na zona ativa de uma fonte de microondas. Como fonte de excitação foi utilizada uma lâmpada de Xe, no modo de operação pulsada. A detecção foi realizada através de uma fotomultiplicadora perpendicular a um feixe de excitação. Aquecendo-se o recipiente, os metais na forma de carbonila foram conduzidos ao atomizador, pelo fluxo de Ar-CO. Os limites de detecção descritos são da ordem de $0,5 \times 10^{-7} \%$ de $(\mathrm{Fe}), 0,05 \times 10^{-7} \%$ de (Ni), $1,0 \times 10^{-7} \%$ de $(\mathrm{Co})$.

Zhang, Li e $\mathrm{Li}^{57}$ descreveram estudos quemométricos para otimização de leituras espectrofotométricas na determinação simultânea de $\mathrm{Co}, \mathrm{Ni}, \mathrm{Cu}, \mathrm{Zn}$ e $\mathrm{Fe}$, usando PAR em meio etanólico e tampão borato. Foram traçados espectros das soluções entre 520-570 nm e suas derivadas calculadas. Os resultados dos picos de cada elemento foram tratados por programa BASIC, para a otimização simplex. A Lei de Beer foi obedecida para quantidades maiores que $12 \mu \mathrm{g} \mathrm{L}^{-1}(\mathrm{Fe})$ e $20 \mu \mathrm{g} \mathrm{L}^{-1}$ $(\mathrm{Co}, \mathrm{Ni}, \mathrm{Cu}, \mathrm{Zn})$, na solução de análise e coeficientes de recuperação de $90-110 \%$ foram observados.

Um método espectrofotométrico computadorizado para estimar constantes de velocidade e concentrações de misturas de componentes foi proposto por Caldera ${ }^{58}$. O sistema químico foi baseado no deslocamento do $\mathrm{Ni}(\mathrm{II})$ e $\mathrm{Co}(\mathrm{II})$, e de seus complexos com o etilenoglicol-bis-(2-aminoetil éter)-NNN'N'-tetraacético, pelo PAR. Os dados espectrofotométricos em função do tempo e do comprimento de onda foram utilizados em cálculos teóricos, resultando em faixas de detecção de $0,1-1 \mu \mathrm{g} \mathrm{mL} \mathrm{m}^{-1}$ para Co e 0,5-5 $\mathrm{g} \mathrm{mL}^{-1}$ para o Ni.

$\mathrm{Hu}$, Xie e Yang ${ }^{59}$ utilizaram o ácido 2-(4,5-dimetil-2tiazolazo)-5-dimetilaminobenzóico, para determinar simultaneamente $\mathrm{Ni}$ (II) e $\mathrm{Co}(\mathrm{II})$ em minerais de níquel em meio etanol/ água, por espectrometria derivativa. A Lei de Beer foi obedecida acima de $0,48 \mathrm{Dg} \mathrm{mL}^{-1}$, dos metais. A interferência de Fe(III) e $\mathrm{Al}(\mathrm{III})$ foi eliminada pela a adição de fluoreto de sódio.

McLaren et al $^{60}$ apresentaram estudos sobre a determinação simultânea de traços de $\mathrm{Fe}, \mathrm{Mn}, \mathrm{Co}, \mathrm{Ni}, \mathrm{Cu}, \mathrm{Zn}, \mathrm{Cd}$ e $\mathrm{Pb}$, em água de mar, utilizando pré-concentração e ICP-MS com 
modificação, para a introdução de amostra por conexão direta ao nebulizador e inclusão de uma coluna de limpeza. Os resultados obtidos foram comparados quanto ao tipo de coluna de préconcentração utilizada, MetPac CC-1 ou I-8-HOQ-8-hidroxiquinolina imobilizada em sílica, com limites de detecção variando entre $1,6 \mathrm{ng} \mathrm{mL}^{-1}(\mathrm{~Pb})$ até $55 \mathrm{ng} \mathrm{L}^{-1}(\mathrm{Ni})$ e $0,3 \mathrm{ng} \mathrm{L}^{-1}$ (Cd) até $47 \mathrm{ng} \mathrm{L}^{-1}(\mathrm{Fe})$, respectivamente para cada coluna. O tempo gasto nas determinações foi de aproximadamente 15 minutos.

Chen, Berndt e Toelg ${ }^{61}$ publicaram resultados de seus estudos sobre detecção simultânea direta, no qual avaliaram o desempenho da espectrometria de absorção atômica de chama, de forno de grafite e ICP com emissão ótica. Os metais analisados, em ligas de ferro de alta pureza, foram $\mathrm{Ba}, \mathrm{Bi}, \mathrm{Cd}, \mathrm{Co}$, $\mathrm{Cr}, \mathrm{Cu}, \mathrm{Mn}, \mathrm{Ni}, \mathrm{Pb}$, Ti e $\mathrm{V}$, com limites de detecção na ordem de $n g \mathrm{~g}^{-1}$. Na determinação direta o melhor desempenho foi obtido pelo ICP, enquanto após a eliminação da matriz de Fe, por extração com solvente, o melhor desempenho foi da EAA com forno de grafite.

Um método para a determinação simultânea de $\mathrm{Cu}, \mathrm{Pb}, \mathrm{Ni}$, $\mathrm{Fe}, \mathrm{Cr}$, Co e Mo, por espectrometria de absorção atômica de chama foi apresentado por Saran et $a^{62}$. Os cátions bivalentes foram complexados utilizando-se 5-(2'-carboxifenil)azo-8quinolinol e, então, extraídos com MIBK. Os coeficientes de variação $(n=10)$, foram entre $2-7 \%$, a recuperação $>95 \%$ e os limites de detecção entre $0,07-0,38 \mu \mathrm{g} \mathrm{mL}^{-1}$, foi verificada interferência de sulfato na determinação de $\mathrm{Pb}$ e fosfato no caso do Mo. Estas interferências foram contornadas pela a adição de Al(III), antes das determinações.

Parkhomenko, Falendish e Pilipenko ${ }^{63}$, propuseram um método espectrofotométrico para a determinação de $\mathrm{Co}(\mathrm{II})$ e Ni(II) utilizando o ácido 4-hidroxi-3-(5-oxo-2-pirazolino-4-ilazo) naftaleno-1-sulfônico. As medidas foram efetuadas em $485 \mathrm{~nm}$, usando-se as diferenças de absortividade molar $(\varepsilon=56600$ para o Co e 42500 para o Ni), os autores descreveram a determinação simultânea dos dois elementos, em uma faixa de concentração entre $0,1-1 \mu \mathrm{g} \mathrm{mL}^{-1}$, com limite de detecção da ordem de $0,1 \mu \mathrm{g} \mathrm{mL} \mathrm{m}^{-1}$.

A determinação simultânea de $\mathrm{Ca}, \mathrm{Mg}, \mathrm{Mn}, \mathrm{Cu}, \mathrm{Zn}, \mathrm{Cd}, \mathrm{Co}$, $\mathrm{Ni}, \mathrm{Pb}$ e $\mathrm{Fe}$ em sais inorgânicos de lítio, usando espectrometria de absorção atômica foi discutida por Shen, Nie e Chen ${ }^{64}$. Os metais foram extraídos para uma fase orgânica (MIBK), à partir de uma solução contendo $\mathrm{NaCl}$, vermelho de fenol, hidróxido de amônio, hexamina e 1-fenil-3-metil-4-benzoilpirazol-5-ona. Uma nova extração com $\mathrm{HCl}, \mathrm{La}(\mathrm{III})$ e sódio, trouxe os metais para a fase aquosa, na qual foram determinados por EAA, em chama de ar-acetileno, recuperando-se entre $94-106 \%$, com limite de detecção da ordem de $0,0001 \%$.

A utilização do algorítmo de filtragem de Kalman, para a resolução de picos de absorção sobrepostos em espectrofotometria, quando se determinam $\mathrm{Co}(\mathrm{II}), \mathrm{Ni}, \mathrm{Zn}$ e $\mathrm{Cd}$ em presença de 5-bromo-2-(2-piridilazo)-5-dietil-aminofenol e do surfactante brometo de hexametilpiridinium, foi descrita por Shi et $\mathrm{al}^{65}$. Os espectros das soluções resultantes foram obtidos entre 500$620 \mathrm{~nm}$ e o tratamento matemático permitiu a determinação simultânea dos quatro metais.

$\mathrm{Ni}$ e Zhang ${ }^{66}$ também descreveram a utilização de um modelo matemático, para a determinação simultânea $\mathrm{Co}(\mathrm{II}), \mathrm{Ni}, \mathrm{Cu}$ (II) e Zn. O método baseia-se na utilização de soluções padrão dos metais, em presença de 2-(5-bromo-2-piridilazo)-5-dietilaminofenol, em diferentes soluções tampão. A detecção da absorbância das soluções entre 490-610 nm forneceu espectros, que foram tratados com auxílio de um programa em linguagem BASIC, baseado no sistema chamado PCA-PLC (análise do componente principal-mínimos quadrados parciais). Os autores não fornecem as figuras de mérito para os resultados obtidos.

Shen, Nie e Chen ${ }^{67}$ apresentaram um procedimento de determinação simultânea de $\mathrm{Pb}, \mathrm{Fe}, \mathrm{Co}, \mathrm{Ni}, \mathrm{Cu}, \mathrm{Mn}, \mathrm{Zn}, \mathrm{Cd}$, todos no estado bivalente, após extração por solvente, utilizando espectrometria de absorção atômica, com chama de ar-acetileno.
O solvente utilizado foi a MIBK e o agente complexante o dietilditiocarbamato de amônio, com recuperação da ordem de 99,4-102,9\%.

Os mesmos autores ${ }^{68}$ descreveram um procedimento semelhante para a determinação simultânea de $\mathrm{Cu}, \mathrm{Cd}, \mathrm{Co}, \mathrm{Ni}, \mathrm{Pb}$ e $\mathrm{Mn}$, na forma bivalente, em sais de metais alcalinos, $\mathrm{LiCl}$, $\mathrm{LiOH}, \mathrm{Li}_{2} \mathrm{CO}_{3}, \mathrm{RbCl}, \mathrm{NaCl}, \mathrm{K}_{2} \mathrm{SO}_{4}$ e $\mathrm{KOH}$, sem verificar interferências. Os cátions foram complexados com PMBP, os complexos extraídos com MIBK e determinados por espectrometria de absorção atômica. O método de adição de padrão forneceu recuperação entre 94-106\%, com limites de detecção da ordem de $0,0001 \%$.

A determinação simultânea de $\mathrm{Co}(\mathrm{II})$, Ni e $\mathrm{Cu}(\mathrm{II})$, utilizando como reagente o PAR, em meio citrato e medindo as absorbâncias das soluções entre 480-530 nm, foi descrita por $\mathrm{Lu}^{69}$. Os metais foram determinados utilizando o método de regressão dos mínimos quadrados parciais, permitindo a previsão dos erros de determinação. Não são fornecidos maiores detalhes, quanto às figuras de mérito.

Utilizando medidas espectrofotométricas tratadas pelo princípio do filtro de Kalman, através de um programa em BASIC, Li et $\mathrm{al}^{70}$ efetuaram a determinação simultânea de $\mathrm{Co}, \mathrm{Ni}, \mathrm{Cu}$, $\mathrm{Zn}$ e $\mathrm{Cd}$, na forma dos cátions bivalentes, em amostras de cabelo. Após abertura, as amostras foram tratadas com NaF, tampão borato $\mathrm{pH}=9,0$ e PAR, em meio aquoso. Os espectros foram obtidos entre $460-580 \mathrm{~nm}$, obtendo-se coeficientes de recuperação entre $93,4-105,9 \%$, quando se utilizaram padrões.

A utilização de procedimentos de análise de regressão linear multi-comprimentos de onda, para a determinação simultânea de $\mathrm{Co}(\mathrm{II})$ e $\mathrm{Ni}(\mathrm{II})$, foi discutida por Blanco et $\mathrm{al}^{71}$. Os autores mediram as absorbâncias de soluções de complexos de cada metal em presença do PAR. O procedimento de cálculo envolveu a razão de absorbância da amostra pela absorbância do padrão em função da absorbância de cada padrão. Os resultados foram linhas retas com interseções e coeficientes angulares proporcionais às concentrações. Segundo os autores o procedimento identifica interferências e corrige efeitos de linhas de base defeituosas.

$\mathrm{Ni}^{72}$ apresentou uma modificação do método dos mínimos quadrados parciais, para determinação simultânea de Co(II), $\mathrm{Fe}(\mathrm{II})$ e $\mathrm{Ni}(\mathrm{II})$, em soluções padrão de metais, utilizando como complexante o 2-(5-bromo-2-piridilazo)-5-dietilaminofenol, em meio borato $(\mathrm{pH}=9,0)$ obtendo espectros entre 500-700 nm.

Kato $^{73}$ estudou um método para a determinação direta de $\mathrm{Fe}$, $\mathrm{Ni}, \mathrm{Cu}, \mathrm{Co}, \mathrm{Mn}$ e $\mathrm{Pb}$ em ligas de zircônio. A matriz de zircônio foi removida por cloração e os metais residuais foram determinados por ICP-EEA, usando curvas analíticas. Os limites de detecção foram da ordem de $\mathrm{ng} \mathrm{L}^{-1}$, dependendo do elemento analisado. O método foi testado em materiais certificados.

A espectroscopia de absorção foi utilizada por $\mathrm{Jin}^{74}$ para determinação simultânea de $\mathrm{Co}, \mathrm{Ni}$ e $\mathrm{Cu}$, em amostras de óxido de tungstênio. Os comprimentos de onda utilizados foram 240,7, 324,7 and 232,0 nm, respectivamente. Os cátions foram previamente complexados com PAR e os complexos separados do tungstênio, por um tampão de amônia em pH=10. Recuperação de $98,4-101,2 \%$ foram relatadas.

$\mathrm{O}$ mesmo autor ${ }^{75}$ estendeu estes estudos avaliando a influência da presença do dodecilbenzenossulfato de sódio, que aumenta a sensibilidade e mascara os efeitos da matriz. A influência do surfactante é aumentar a eficiência da atomização, segundo o autor.

Um estudo semelhante ao da referência 68 , porém utilizando como complexante o dietilditiocarbamato de amônio, em meio acetato $(\mathrm{pH}=5,7)$, foi descrito por Shen, Chen e $\mathrm{Nie}^{76}$.

A determinação simultânea de $\mathrm{Ni}(\mathrm{II}), \mathrm{Cu}$ (II) e $\mathrm{Co}(\mathrm{III})$, utilizando ácido 5-(sulfometilamino)-2-(2-tiazilazo)-p-toluico (I), foi descrita por Zhao e Feng ${ }^{77}$. As absorbâncias da amostra em presença de tampão acetato $(\mathrm{pH}=5,5)$ e iodato de potássio, além do reagente I, foram medidas em 551, 588 e $655 \mathrm{~nm}$. A Lei de 
Beer foi obedecida para $30 \mu \mathrm{g} / 25 \mathrm{~mL}$, de cada complexo. Os autores afirmam que os resultados foram satisfatórios.

$\mathrm{Li}$, Peng e $\mathrm{Li}^{78}$ apresentaram um procedimento para determinação de $\mathrm{Co}(\mathrm{II}), \mathrm{Ni}(\mathrm{II}), \mathrm{Zn}(\mathrm{II})$ e $\mathrm{Cd}(\mathrm{II})$, utilizando 2-(5bromo-2-piridilazo)-5-dietilaminofenol, como complexante, em meio amoniacal $(\mathrm{pH}=8,0)$ e em presença de brometo de hexadecilpiridinium. A absorbância das soluções foi medida entre 500-620 nm e submetida a tratamento matemático por filtro de Kalman, através de um programa em BASIC. Os gráficos de calibração foram lineares acima de $8 \mu \mathrm{g} / 25 \mathrm{~mL}$ de Co, $\mathrm{Cu}$, Ni e Zn e acima de $10 \mu \mathrm{g} / 25 \mathrm{~mL}$ de Cd. Recuperações de 95-105\% foram obtidas, sem interferências.

Resultados sobre a determinação simultânea de traços de $\mathrm{Ag}, \mathrm{Cd}, \mathrm{Li}, \mathrm{Co}$ e Ni, em amostras geológicas foram apresentados por Gong ${ }^{79}$. Após a abertura das amostras, em uma mistura de ácidos, estas foram diluídas e adicionou-se KI, ácido ascórbico e $\mathrm{HCl}$, sendo finalmente extraídas com MIBK. A prata e o cádmio na fase aquosa e o cobalto e níquel da fase orgânica foram determinados por EAA e o Li por EEA. Os limites de detecção foram 0,02 (Ag e Cd), 2,4(Li) 1,2 (Co) e $1,9 \mathrm{ppm}(\mathrm{Ni})$. Os coeficientes de variação foram função dos metais analisados e suas concentrações.

A determinação simultânea de misturas de $\mathrm{Co}(\mathrm{III})-\mathrm{Cu}(\mathrm{II})$ e $\mathrm{Co}(\mathrm{III})-\mathrm{Ni}(\mathrm{II})$, baseado na diferença de velocidade de formação de seus complexos com 3-(1-H-1,2,4-triazol-3-ilazo)tolueno-2,6-diamina, foi proposta por Arias, Jimenez e Jimenes ${ }^{80}$. O método é baseado no fato de que os complexos de Co tem formação muito mais lenta que os demais, medindose sua absorbância, após a determinação do $\mathrm{Ni}$ e $\mathrm{Cu}$. Os erros e desvios padrão são descritos para cada íon.

Ping $^{81}$ estudou a determinação simultânea de Co e Ni, utilizando como reagente complexante o [2-(5-bromo-2piridilazo)-5-dimetilaminofenol] (5-Br-PADAP). Neste caso as absorbâncias em 520, 530, 540, 550, 560, 570, 580 e $590 \mathrm{~nm}$ foram medidas, após mistura da amostra com o 5-Br-PADAP, ácido tartárico e ácido pirogálico (como mascarante para $\mathrm{Fe}$ ), em meio amoniacal $(\mathrm{pH}=10)$. Os resultados foram tratados pelo método dos mínimos quadrados. Recuperações de 93-103\% (Co) e $98-104 \%(\mathrm{Ni})$, foram relatadas.

$\mathrm{Li}$, Chen e $\mathrm{Yi}^{82}$ desenvolveram estudos de determinação espectrofotométrica simultânea de $\mathrm{Co}$ e $\mathrm{Ni}$, em meio de tartarato duplo de sódio e potássio, timolftaleína, tampão amônia, tiouréia, dodecilbenzenossulfonato e diaminoazobenzeno. As medidas de absorbância em $700 \mathrm{~nm}$, foram relacionadas com a concentração do cobalto, enquanto os picos em $620 \mathrm{~nm}$ foram utilizados para calcular a concentração de níquel, após descontar-se a contribuição do Co, neste comprimento de onda. Descreveram-se erros relativos de $0,4 \%(\mathrm{Co})$ e $0,5 \%(\mathrm{Ni})$. Foi observada a interferência do cobre.

Um procedimento para determinação simultânea de $\mathrm{Cu}$, Co e $\mathrm{Ni}$, utilizando o PAR como complexante em $\mathrm{pH}=9,5$, foi apresentado por Lu e Shi ${ }^{83}$. As absorbâncias foram determinadas entre $460-560 \mathrm{~nm}$, a cada $5 \mathrm{~nm}$. Os resultados obtidos foram tratados matematicamente.

Estudos sobre as condições ideais para determinação simultânea de $\mathrm{Co}, \mathrm{Ni}, \mathrm{Fe}$ e $\mathrm{Cu}$, com EDTA, utilizando método matemático para tratamento de dados espectrofotométricos, foi descrito por $\mathrm{Ke}, \mathrm{Yu}$ e Shiao ${ }^{84}$.

Um procedimento para determinação espectrofotométrica de $\mathrm{Ni}$ e Co, usando procedimento de duplo-comprimento de onda, foi relatado por Cheng e $\mathrm{Li}^{85}$. O meio reacional utilizado foi fluoreto de sódio e 4,4'-dipiridila, brometo de hexadeciltrimetilamônio e eriocromo azurol-B, como complexantes. O tratamento dos dados foi realizado descontando-se as absorbâncias em 619 e 634 nm (dos complexos de Ni e Co, respectivamente), as absorbâncias em 642 e $598 \mathrm{~nm}$ foram usadas como referência. A Lei de Beer foi obedecida acima de 0,4 (Ni) e acima de $0,44 \mu \mathrm{g} \mathrm{mL} \mathrm{mL}^{-1}$ (Co).

Lopes et $\mathrm{al}^{86}$ efetuaram determinações simultâneas de Co e
$\mathrm{Ni}$, resolvendo picos sobrepostos por espectrofotometria derivativa entre 400 e $600 \mathrm{~nm}$. Utilizando 2-piridilcetona e 2quinolinohidrazona, os comprimentos de onda medidos foram 480 e $512 \mathrm{~nm}$, respectivamente. Os autores relataram erros de até $10 \%$, em concentrações da ordem de $\mu \mathrm{g} \mathrm{mL} \mathrm{m}^{-1}$.

Diferenças no comportamento químico de complexos de Ni e $\mathrm{Co}$, foram utilizadas por Fang e Zhao ${ }^{87}$ para a sua determinação simultânea. O método consistiu na separação dos etilxantatos em $25 \mathrm{~mL}$ de acetato de etila, na presença de tartarato amônio e acetato de sódio. A absorbância da fase orgânica foi medida, sendo relacionada com a soma da absorbância relativa ao $\mathrm{Ni}$ e Co, em $410 \mathrm{~nm}$. A adição de ácido perclórico, levou à destruição do complexo Ni-etilxantato. Mediu-se então, a absorbância relativa ao $\mathrm{Co}$ e, por diferença a de $\mathrm{Ni}$. Curvas analíticas foram lineares acima de $400(\mathrm{Co})$ e $700 \mu \mathrm{g} / 25 \mathrm{~mL}(\mathrm{Ni})$, com recuperações de 98,6 e $106 \%$, respectivamente.

Uma comparação de métodos computacionais, na resolução de espectros de misturas de $\mathrm{Cu}(\mathrm{II}), \mathrm{Co}(\mathrm{II})$ e $\mathrm{Ni}(\mathrm{II})$, complexados com 2-(5-bromo-2-tiazoilazo)-5-dietilaminofenol (5-Br-TADAP), foi apresentada por Wang, Zhou e Luo ${ }^{88}$.

Kane $^{89}$ descreveu um estudo sobre a otimização de parâmetros de chama para determinação simultânea de $\mathrm{Co}, \mathrm{Cu}, \mathrm{Mn}$, $\mathrm{Ni}, \mathrm{Pb}, \mathrm{Zn}, \mathrm{Ag}$ e $\mathrm{Cd}$, por EAA, variando posição de leitura e testando diferentes relações ar/acetileno. As conclusões foram baseadas nas recuperações e limites de detecção calculados. A razão ar/acetileno $1: 5,9$, com leituras $5 \mathrm{~mm}$ acima do queimador, foram as condições com melhor desempenho.

A técnica de espectrofotometria derivativa, foi também utilizada por Murillo et al ${ }^{90}$ para determinação de Co e Ni, utilizando como agente complexante 1-hidroxiantraquinona-2-ácido carboxílico. A determinação de Co, foi efetuada no ponto em que a primeira derivada passa pelo zero no comprimento de onda do $\mathrm{Ni}(510,5 \mathrm{~nm})$, enquanto o $\mathrm{Ni}$ foi determinado no zero de Co (494,5 $\mathrm{nm}$ ), determinando-se Co entre $0,75-4,5 \mu \mathrm{g} \mathrm{mL}-1$ e $\mathrm{Ni}$ entre $0,50-3,0 \mu \mathrm{g} \mathrm{mL}^{-1}$. Histogramas de erros e avaliação dos limites de confiança foram apresentados.

$\mathrm{Li}, \mathrm{Xi}$ e $\mathrm{Shi}^{91}$ mediram os espectros de complexos de Co e $\mathrm{Ni}$ com 2-(5-bromo-2-piridilazo)-5-dimetilaminofeno, entre 500-650 nm. Os resultados foram tratados usando métodos computacionais, para cálculo das concentrações de Co e Ni. Relações lineares foram obtidas acima de 24 (Co) e $15 \mu \mathrm{g} / 25$ $\mathrm{mL}(\mathrm{Ni})$, sendo o método aplicável para relações $\mathrm{Co} / \mathrm{Ni}$ entre 20:1 e $1: 15$.

$\mathrm{Ni}^{92}$ apresentou um procedimento matemático de cálculo, baseado na medida de absorbâncias do ligante e de região espectral cobrindo a faixa de picos de complexos metálicos. Os cálculos foram efetuados por sistema matricial e o método foi aplicado a sistemas contendo $\mathrm{Cu}, \mathrm{Ni}$ e $\mathrm{Co}$ ou $\mathrm{Cu}, \mathrm{Ni}$, Co e $\mathrm{Zn}$, complexados com 2-(5-bromo-2-piridilazo)-5-dietilaminofenol. A absorbância do ligante foi medida em $430 \mathrm{~nm}$ e as dos complexos metálicos entre $510-590 \mathrm{~nm}$, com intervalos de $10 \mathrm{~nm}$. Para concentrações entre $1-5 \mu \mathrm{g} / 25 \mathrm{~mL}$ de cada componente metálico, o autor considerou os resultados satisfatórios.

Klaentschi, Esenwein e Mueller ${ }^{93}$ determinaram Si, Mn, P, $\mathrm{Cu}, \mathrm{Al}, \mathrm{Ni}, \mathrm{Cr}, \mathrm{Mo}, \mathrm{V}, \mathrm{Ti}, \mathrm{Co}$ e As, em aços, utilizando ICPEEA. As amostras foram abertas e tratadas com peroxidissulfato de amônio. Foram selecionados comprimentos de onda adequados, obtendo-se limites de detecção de 0,0003-0,015\%, de metal no aço.

O reagente 1-tiobenzoil-3-p-toluiltiouréia, foi utilizado por Ambhore e Joshi ${ }^{94}$, na determinação espectrofotométrica de Ni e Co. Os complexos formados foram extraídos com benzeno e mediu-se a absorbância da fase orgânica em 405-465 nm, para $\mathrm{Co}$ e Ni respectivamente. As regiões lineares foram respectivamente $0,2-1,6$ e $0,5-4 \mu \mathrm{g} \mathrm{mL}{ }^{-1}$. Interferência de $\mathrm{Cu}$ foi eliminada pela precipitação do cátion na forma de sulfeto.

Um procedimento semelhante ao da referência 85, para determinação simultânea de $\mathrm{Co}$ e $\mathrm{Ni}$, foi descrito por Luo e Yang ${ }^{95}$. Neste caso a técnica utilizada foi a espectrofotometria 
com duplos comprimentos de onda, o complexante foi o PAR, em meio citrato $(\mathrm{pH}=9)$, sendo os metais determinados pela diferença de absorbância em 515/480 e 498/531 nm (Ni/Co). As curvas analíticas foram lineares acima de $60 \mathrm{Dg} / 50 \mathrm{~mL}$ para os dois cátions.

A determinação espectrofotométrica simultânea de Co, Ni e $\mathrm{Cu}$ em óxidos de tungstênio e molibdênio, foi apresentada por Luo, $\mathrm{Wu}$ e $\mathrm{Wu}^{96}$. O método foi baseado nos complexos dos metais com 1-(2-piridilazo)-2-naftol, com máximos de absorção em 550(Cu), 560(Ni) e 580(Co), com Lei de Beer obedecida acima de $0,5 \mu \mathrm{g} \mathrm{mL}^{-1}$. O método se mostrou útil para determinações na faixa de 4-80(Cu), 5-200(Ni) e 11-180 ppm (Co).

Rios e Valcarcel ${ }^{97}$ utilizaram-se da diferença de velocidade de reação de troca de grupos $\mathrm{C}=\mathrm{N}$ entre tiosemicarbazida e 6metilpicolinaldeído azina, em presença de $\mathrm{Cu}, \mathrm{Co}$ e $\mathrm{Ni}$, em meio acetato. A absorbância das soluções, em $410 \mathrm{~nm}$, foi acompanhada em função do tempo e equações foram desenvolvidas para o tratamento dos dados obtidos. Misturas binárias apresentaram coeficientes de variação $(n=22)$, entre $0,4-2,3 \%$, nas concentrações utilizadas no estudo. Interferências também foram discutidas.

Misturas binárias de $\mathrm{Ni}(\mathrm{II}), \mathrm{Co}(\mathrm{III})$ e $\mathrm{V}(\mathrm{V})$, foram determinadas espectrofotometricamente utilizando-se como complexante o ácido 3(picolideno)- benzenossulfônico[ $\alpha$-(3-sulfofenil) picolinaldeído]-2-hidroxibenzoilhidrazona, por Garcia-Vargas, et $\mathrm{al}^{98}$. Os comprimentos de onda dos máximos de absorção foram 375 e $385 \mathrm{~nm}$ (Ni), 400 e 415 (Co) e $395 \mathrm{~nm}$ (V). Mascarantes para $\mathrm{V}$ e $\mathrm{Cu}$ foram utilizados, quando estes estavam presentes nas misturas. Os autores descrevem detalhadamente os resultados obtidos, em função das misturas preparadas ou dos interferentes testados.

Determinações de Co e Ni por espectrofotometria após extração com tetracloreto de carbono, dos complexos destes metais com etiltioxantato, foram realizadas por Rao e Shekar ${ }^{99}$. As absorbâncias dos complexos foram determinadas em 389 (Co) e $495 \mathrm{~nm}(\mathrm{Ni})$, com linearidade da Lei de Beer acima de 3,5 e $7 \mu \mathrm{g} \mathrm{mL}{ }^{-1}$, respectivamente. Foi observado que $\mathrm{Cu}(\mathrm{II})$, $\mathrm{Bi}(\mathrm{III})$ e $\mathrm{Fe}$ (III), interferem. Teste do método em material certificado mostrou coeficiente de variação de 0,5-1\%.

Nesterenko e Ivanov $^{100}$ apresentaram a espectrofotometria convencional e derivativa na determinação simultânea de $\mathrm{Au}(\mathrm{III})$, metais platínicos [Pt(IV), Pd(II), $\mathrm{Rh}(\mathrm{II})$ e $\mathrm{Fe}(\mathrm{III})]$ e não ferrosos [Cu(II), Ni(II), Co(II) e $\mathrm{Mn}(\mathrm{II})]$. O método foi baseado na medida dos espectros de misturas binárias ou terciárias dos elementos acima, em meio de ácido sulfúrico / brometo de potássio, entre 360 e $450 \mathrm{~nm}$ (com intervalos de 10 nm) e tratamento matemático dos resultados. A solução de referência, "branco", consistiu de AuBr ${ }^{4-}$. Descrevem-se as quantidades relativas de cada metal, não se observando interferência de $\mathrm{Zn}, \mathrm{Pb}(\mathrm{II})$ e $\mathrm{In}(\mathrm{III})$, respectivamente até $6.000,1.000$ e 210 vezes mais concentrados que o ouro.

A formação de complexos de $\mathrm{Fe}(\mathrm{II}), \mathrm{Co}(\mathrm{II})$ e $\mathrm{Ni}(\mathrm{II})$, com bis-2-piridilcetonapirimidina-2-ilhidrazona, foi utilizada por Desmukh ${ }^{101}$ para a determinação simultânea destes cátions. As medidas nos máximos de absorbância de cada espécie (580, 460 e $430 \mathrm{~nm}$, respectivamente), foram utilizadas para montar sistemas de equações, que permitiram obter as concentrações de cada componente. O método se mostrou aplicável para 0,4 $4(\mathrm{Fe}), 0,1-2(\mathrm{Co})$ e $0,1-1 \mu \mathrm{g} \mathrm{mL}^{-1}(\mathrm{Ni})$. São discutidas as interferências em cada sistema.

Wasey, Bansal e Satake ${ }^{102}$ descreveram a determinação de Co e Ni, individual e simultaneamente, através da extração de seus complexos com fenantrenoquinona monoxima, por naftaleno fundido. Após solidificação, a fase do naftaleno foi dissolvida com DMF e as absorbâncias em 460 (Ni) e $470 \mathrm{~nm}$ (Co), foram determinadas e relacionadas com a concentração do metal entre $1,2-10,6$ e $0,6-7,8 \mathrm{Dg} / 10 \mathrm{~mL}$, respectivamente.

McLaren et al ${ }^{103}$ determinaram simultaneamente diversos metais utilizando ICP-EEA, em sedimentos marinhos. Os metais foram divididos em dois grupos chamados majoritários (Al, Fe, $\mathrm{Ca}, \mathrm{Mg}, \mathrm{Na}$ e $\mathrm{P}$ ) e minoritários (Be, $\mathrm{Co}, \mathrm{Cu}, \mathrm{Mn}, \mathrm{Ni}, \mathrm{Pb} \mathrm{V}$ e Zn), cada grupo analisado sob condições experimentais adequadas. Descreveram-se procedimentos para correções espectrais e as linhas utilizadas na determinação de cada elemento. Limites de detecção de 0,05-5 $\mu \mathrm{g} \mathrm{g}^{-1}$ foram descritos para duas amostras.

Savinova et al $^{104}$ descreveram a determinação simultânea de $\mathrm{Cu}, \mathrm{Co}, \mathrm{Mo}, \mathrm{Mn}$, Ni e V, em plantas por espectrofotometria usando um preparado sólido, composto por uma mistura de grafite, carbonato de bário e óxido de gálio. Os limites de detecção descritos foram $4.10^{-5}, 5.10^{-5}, 5.10^{-5}, 1.10^{-4}, 1.10^{-4}$, $1.10^{-4} \%$, respectivamente.

A utilização de 1-fenil-3-tiobenzoiltiocarbamida, como reagente para a determinação de $\mathrm{Ni}$ e Co, após extração dos complexos com clorofórmio, foi descrita por Ilyas e Joshi ${ }^{105}$. $\mathrm{O}$ complexo de Co apresentou cor laranja, com máximo de absorção em $400 \mathrm{~nm}$ e o de Ni cor amarela, com máximo em $460 \mathrm{~nm}$. As regiões lineares de resposta foram entre 0,13$1,33 \mathrm{Dg} \mathrm{mL} \mathrm{mL}^{-1}$ para o Co e $0,66-3,61 \mathrm{Dg} \mathrm{mL} \mathrm{mL}^{-1}$ para o $\mathrm{Ni}$

$\mathrm{O}$ reagente 2-[4-amino-3-(1,2,4-triazoilazo)]-naftol-4sulfonato de sódio, foi utilizado por Mukherjee et al ${ }^{106}$, na determinação espectrofotométrica simultânea de $\mathrm{Ni}$ (II) e Co(II), em óleos vegetais hidrogenados. Gráficos de absorbância em função da concentração, foram lineares para concentrações acima de 2,06 (Co) e 2,76 ppm (Ni).

A utilização de hexametilfosforamida (HMPA), como reagente para determinação de $\mathrm{Co}, \mathrm{Ni}$ e $\mathrm{Fe}$ por espectrofotometria, na presença de tiocianato, foi descrita por Bruno et $\mathrm{al}^{107}$. Os máximos de absorção foram observados entre $618 \mathrm{e}$ $317(\mathrm{Co}), 482$ e $317(\mathrm{Fe})$ e 600 e $800 \mathrm{~nm}(\mathrm{Ni})$. Mudança de $\mathrm{pH}$ entre 3-10, não alterou a forma dos espectros. Os íons de $\mathrm{Mg}$, $\mathrm{Al}, \mathrm{Zn}, \mathrm{Mn}, \mathrm{Ca}$ e $\mathrm{Ba}$, não interferiram até $1.10^{-3} \mathrm{~mol} \mathrm{~L}^{-1}$. Para a relação $\mathrm{C}_{\mathrm{Ni}} / \mathrm{C}_{\mathrm{Co}}$ até 10 não se observou interferência entre as duas espécies e o limite de detecção foi de $10^{-6} \mathrm{~mol} \mathrm{~L}^{-1}$.

\section{FIA}

Maksimova e Morosanova ${ }^{108}$ propuseram um método, no qual a amostra após ser misturada com um tampão borato ou citrato $\mathrm{pH}=5,85$, tem seu fluxo dividido em dois. Um deles passa por uma coluna de $40 \mathrm{mg}$ de Diasorc-C16, modificada pela passagem prévia de $6 \mathrm{~mL}$ de solução de a-nitroso-b-naftol, $0,5 \mathrm{mmol} \mathrm{L} \mathrm{L}^{-1} \mathrm{em}$ água etanol 19:1 (v/v), em um fluxo de $482 \mu \mathrm{L} \mathrm{min}^{-1}$, para remoção do Co. O fluxo é, então, segmentado com ar e finalmente recebe uma solução de PAR. A absorbância do complexo formado com o Ni é acompanhada em $505 \mathrm{~nm}$. A segunda porção do fluxo recebe o PAR, sem separação dos metais e sua absorbância, somatório das absorbâncias dos dois complexos, é medida em 505 $\mathrm{nm}$. Este procedimento foi aplicado, pelos autores, a amostras contendo entre $2-80 \mu \mathrm{mol} \mathrm{L} \mathrm{L}^{-1}$ de $\mathrm{Co} . \mathrm{Cu}, \mathrm{Ni}, \mathrm{Fe}$ ou $\mathrm{Cd}$, não interferiram em concentrações 10 vezes maiores.

Arruda, Zagatto e Maniasso ${ }^{109}$ propuseram um método para determinação simultânea de $\mathrm{Co}$ e Ni, no qual a amostra transportada por um fluxo carregador de $\mathrm{HCl}$ foi misturada com tetraborato de sódio. O fluxo resultante foi tratado com citrato de sódio e PAR, usando-se um reator na forma de bobina, após o que são adicionados EDTA e pirofosfato de sódio, como mascarantes. Detecção espectrofotométrica a $520 \mathrm{~nm}$, mostra a absorbância relativa ao somatório das absorbâncias dos complexos de Co e Ni, quando se usa uma bobina de $400 \mathrm{~cm}$. Quando se utiliza o dobro da amostra e uma bobina de $1 \mathrm{~cm}$, o sinal a $520 \mathrm{~nm}$ se deve apenas à presença do complexo de Co, já que a decomposição do complexo $\mathrm{Ni}$ /citrato é muito mais lenta que a do $\mathrm{Co} /$ citrato. $\mathrm{O}$ método foi testado em aços, nas faixas de concentração 2-5,24 mg L ${ }^{-1}$ de Co e $0,12 \mathrm{mg} \mathrm{L}^{-1}$ de Ni, com RSD $(\mathrm{n}=5), 3,1 \%$ e $0,9-7 \%$, respectivamente.

Rieder e Noergaard ${ }^{110}$ utilizaram o reagente PAR, para determinação simultânea de $\mathrm{Ni}$ e Co. Os reagentes foram misturados em um fluxo carregador de água e tamponados em pH=9. 
Tomaram-se medidas de 51 comprimentos de onda e 61 diferentes tempos de leitura. Soluções padrão foram selecionadas por calibração multivariada. Detectou-se interferência de $\mathrm{Zn}$ e $\mathrm{Cu}(\mathrm{II})$. A determinação dos metais principais foi conseguida com erro de $3-4 \%$.

Utilizando-se da diferença da velocidade de formação dos complexos de 5-(octiloximetil)-quinolina com $\mathrm{Ni}$ (II) e $\mathrm{Co}$ (II). O complexo de $\operatorname{Co}($ II) tem velocidade de formação 44 vezes maior, em meio surfactante não miscelar, Tagashira et al $^{111}$ determinaram as duas espécies. As amostras foram misturadas com Triton X-100, em tampão fosfato $\mathrm{pH}=7$. A mudança da absorbância foi acompanhada em $380 \mathrm{~nm}$, acima de 3-4 minutos. Curvas analíticas se mostraram lineares entre $2-50 \mu \mathrm{mol} \mathrm{L}{ }^{-1}$, com coeficientes de recuperação de $97-100 \%$.

Yamane e Ishimizu ${ }^{112}$ também se utilizaram da diferença de velocidade de substituição do ácido 3-[3-amino-4-(5-bromo-2piridilazo)-N-propilamino] propanossulfônico, por ácido nitriloacético, em complexos de $\mathrm{Co}$ e $\mathrm{Ni}$, para sua determinação. A velocidade de substituição é 100 vezes maior no caso do Ni que no caso do Co. Após a mistura da amostra com o primeiro complexante, esta passa por uma bobina de reação, em seguida recebe o fluxo do ácido nitriloacético e uma válvula para a zona reativa dentro da célula de fluxo, quando então, se mede a absorbância da mistura em $580 \mathrm{~nm}$. Os coeficientes de variação $(n=7)$, foram 1,9 e $2,1 \%$, para Ni e Co respectivamente, com velocidade analítica de 10 análises $\mathrm{h}^{-1}$.

Um procedimento de análise simultânea de $\mathrm{Ni}$ e Co em águas naturais e amostras de rins bovinos foi apresentado por Eskilsson, Haraldson e Jagner ${ }^{113}$. O tratamento prévio das amostras envolveu digestão e redução de Co(III) a Co(II), com borohidreto de sódio. Os metais foram analisados na forma de complexos de DMG, em sistema de fluxo por cronopotenciometria de redissolução catódica, em cela de fluxo de camada fina, equipada com um eletrodo indicador de carbono vítreo/ $\mathrm{Hg}$ e ECS como referência. Um sistema microprocessado foi desenvolvido para controlar as etapas de deposição do $\mathrm{Hg}$ sobre o eletrodo indicador, as quais foram: a) deposição potenciostática a $-0,5 \mathrm{~V}$, b) redução amperostática com registro simultâneo da curva potencial-tempo, c) remoção química do filme de $\mathrm{Hg}$, d) limpeza do eletrodo. Utilizando-se $70 \mathrm{~s}$ para adsorção, observaram-se limites de detecção de 8,6 (Ni) e $10,5 \mathrm{ng} \mathrm{L}^{-1}(\mathrm{Co})$.

Comparação entre três arranjos de sistema por injeção em fluxo foram testados por Fernandez, Luque-de-Castro e Valcarcel ${ }^{114}$ para a determinação simultânea de $\mathrm{Ni}$ e Co, na faixa de $0,1-1 \mathrm{mmol} \mathrm{L}^{-1}$. As determinações foram baseadas na diferença de velocidade de formação dos complexos destes metais com 2-hidroxibenzaldeído tiosemicarbazona. Descreveram-se os diagramas de montagem, concluindo-se qual o melhor com base nos resultados obtidos.

Betteridge e Fields ${ }^{115}$ descreveram um sistema FIA, para determinação simultânea de $\mathrm{Co}(\mathrm{II})$ e $\mathrm{Ni}(\mathrm{II})$, aproveitando-se da diferença de velocidade de decomposição dos citrato complexos em $\mathrm{pH}=9,5$. As absorbâncias dos complexos de PAR, que apresentam formação rápida, foram acompanhadas. Verificouse interferência de $\mathrm{Cu}(\mathrm{II}), \mathrm{Mn}(\mathrm{II}), \mathrm{Zn}$ (II), Cr(III), $\mathrm{Pb}$ (II) e V(V), em concentrações de 10 ppm. Níveis de detecção da ordem de ppm foram obtidos, com velocidade analítica de até 30 analises $\mathrm{h}^{-1}$, com erros menores que $10 \%$.

\section{OUTROS MÉTODOS OU MÉTODOS MISTOS}

Buldini, Ferri e Nobili ${ }^{116}$ apresentaram estudos comparativos, entre a espectrometria de absorção atômica com forno de grafite e a voltametria de redissolução anódica, na determinação simultânea de cátions metálicos. Para determinação de Mn, Co e $\mathrm{Ni}$, os autores utilizaram meio formado por tampões fosfato e citrato. As conclusões foram de que as técnicas apresentaram sensibilidades semelhantes, porém apesar de mais lenta, a voltametria apresenta melhor precisão, com menor número de experimentos, assim suas vantagens são discutidas.

A determinação de $\mathrm{Al}, \mathrm{Cd}, \mathrm{Cr}, \mathrm{Co}, \mathrm{Cu}, \mathrm{Fe}, \mathrm{Pb}, \mathrm{Mn}, \mathrm{U}, \mathrm{V}$ e $\mathrm{Zn}$, em águas naturais foi proposta pelo "Department of the Enviroment (UK)" "117, utilizando voltametria de redissolução e EAA. Segundo a metodologia apresentada, pode-se determinar $\mathrm{Cu}, \mathrm{V}, \mathrm{Zn}$ e $\mathrm{Al}$, por voltametria de redissolução de pulso diferencial com EGPM. U e Fe podem ser determinados com voltametria de redissolução catódica de varredura linear. Determinações simultâneas de $\mathrm{Cd} / \mathrm{Pb}$ e $\mathrm{Ni} / \mathrm{Co}$ foram efetuadas por voltametria de redissolução de pulso diferencial anódica e de varredura linear catódica, respectivamente. Em águas salinas determinaram-se $\mathrm{Cd}, \mathrm{Cu}, \mathrm{Pb}$, Ni e $\mathrm{Zn}$, na forma de ditiocarbamatos, por EAA. Análises de $\mathrm{Cr}$, por EAA eletrotérmica, foram descritas.

\section{CONCLUSÃO}

Com base na quantidade de trabalhos apresentados pode-se afirmar que o tema é de interesse e atualidade, dentro da química analítica.

No caso da cromatografia destacam-se as aplicações em CLAE, usando-se diferentes complexantes, fases estacionárias e detectores.

Em eletroanalítica técnicas amperométricas são largamente utilizadas com destaque para os métodos de análise na modalidade de redissolução. Os métodos nem sempre são de determinação direta, havendo necessidade de tratamento matemático dos resultados obtidos.

Os métodos espectrométricos de análise mais utilizados foram a espectroscopia de absorção molecular, com tratamento matemático, derivativo ou deconvolutivo dos espectros obtidos para separação dos picos. As técnicas de absorção atômica e plasma, também foram amplamente utilizadas.

Métodos automatizados baseados em sistemas de injeção em fluxo foram também descritos, sendo a maioria destes baseados nas diferenças de velocidade de formação de complexos de $\mathrm{Co}$ e $\mathrm{Ni}$, com diversos complexantes.

\section{GLOSSÁRIO DE SÍMBOLOS E ABREVIATURAS}

ac- corrente alternada

CLAE- cromatografia líquida de alta eficiência

dc- corrente contínua

DMF- dimetilformamida

DMG- dimetilglioxima

DPTH- 1,5-bis(di-2-piridilmetileno tiocarbonohidrazina)

EDTA- ácido etilenodiaminotetraacético

ECS- eletrodo de calomelano saturado

EDA- etilenodiamina

EGM- eletrodo gotejante de mercúrio

EGPM- eletrodo de gota pendente de mercúrio

EEA- espectroscopia de emissão atômica

EAA- espectroscopia de absorção atômica

EAA-FG- espectroscopia de absorção atômica, com forno de grafite

FIA- análise por injeção em fluxo

Hz- hertz

ICP- espectroscopia de plasma induzido

ICP-EEA- espectroscopia de emissão atômica, com plasma induzido

ICP-MS- espectroscopia de plasma induzido, acoplada a espectrometria de plasma

MIBK- metil-isobutilcetona

PAR- 4-(2-piridil)azoressorcinol

PADAP- 2-piridilazo-5-dimetilaminofenol

PCA-PLC- método de análise do componente principal mínimos quadrados parciais 
PMBP- 1-fenil-3-metil-4-benzilpirazolona

ppm- partes por milhão

ppb- partes por bilhão

PTFE- teflon

RSD- desvio padrão relativo

s- segundos

SSS- sulfossalicilato de sódio

SPGRMLR- programa para tratamento de dados

SPGRFSQ- programa para tratamento de dados

TAC- 2-(2-tiazoilazo)-p-cresol

TADAP- tiazoilazo-5-dietilaminofenol

THF - tetrahidrofurano

UV- ultra-violeta

V- volts

\section{REFERÊNCIAS}

1. Antunes, P. A.; Cavalheiro, E. T. G.; Chierice, G. O.; Livro de Resumos IX Euroanalysis, Mo P133.

2. Dolezal, J.; Sommer, F.; Collect. Czech. Chem. Commum. 1994, 59, 2209.

3. Rodriguez, V.G.; Romero, J. M. C.; Solis, J. M. F.; Iglesias, J. P.; Lago, H. M. S.; J. Chromatogr.-A 1994, 673, 291.

4. Rodriguez, V. G.; Romero, J. M. C.; Solis, J. M. F.; Iglesias, J. P.; Lago, H. M. S.; Anal. Lett. 1994, 27, 1399.

5. Sturaro, A.; Parvoli, G.; Doretti, L.; Zanchetta, S.; Allegri, G.; Battiston, G. A.; Anal. Chim. Acta. 1993, 274, 163.

6. Li, L. J.; Gui, M. D.; Zhou, Z. F.; Zhao, Y. Q., FenxiShiyansshi 1993, 12, 27; apud Anal. Abstracts, 5511D111.

7. Miura, J.; Tokunaga, N.; Nippon Kagaku Kaishi 1993, 177; apud Anal. Abstracts, 5511D023.

8. Li, C.; Fan, B.; Jiang, B. Fenxi-Shiyansshi 1992, 11, 27; apud Anal. Abstracts, 5501D146.

9. Wang E.; Liu, A; Fenxi-Huaxue 1991, 19, 869; apud Anal. Abstracts, 5409D014.

10. Ou, Y.; Fu, H.; Huang; C.; Fenxi-Huaxue 1990, 18, 945; apud Anal. Abstracts, 5403D015.

11. Xie, N.; Huang, C. e Fu, H.; Sepu 1990, 8, 114; apud Anal. Abstracts, 5303D009.

12. Chen, C.; Guo, H.; Lihua-Jianyan, Huaxue-Fence 1989 , $25,28$.

13. Ji, H.; Cao, S.; Wang, E.; Sepu 1988, 6, 36; apud Anal. Abstracts, 5009D120.

14. Irth, H.; De-Jong, G. J.; Brinkman, U. A. T.; Frei, R. W.; Anal. Chem. 1987, 59, 98

15. Roston, D. A.; Anal. Chem. 1984, 56, 241

16. Bond., A. M.; Wallace, G. G.; Anal. Chem. 1984, 56, 2085.

17. Bond., A. M.; Wallace, G. G.; J. Liq. Chromatogr. 1983, 6, 1799.

18. Bond., A. M.; Wallace, G. G.; Anal. Chem. 1982, 54, 1706.

19. Ichinoki, S.; Morita, T.; Yamazaki, M.; J. Liq. Chromatogr. 1983, 6, 2079.

20. Vukomanovic, D. V.; Page, J. A.; vanLoon, G. W.; Anal. Chem. 1996, 68, 829.

21. Zhou, L. J.; Yu, J. M.; Li, H. M.; Bi, S. W.; Zhou, Q. Z.; Zhang, S. F.; Fenxi Huaxue 1995, 23, 685; apud Anal. Abstracts, 5711H076.

22. Paneli, M. G.; Voulgaropoulos, A. N.; Fresenius'-J.Anal. Chem. 1994, 348, 837.

23. Wang, Y. Q.; Zhao, K. I.; Jiao, K; Fenxi Huaxue 1994, 22, 175; apud Anal. Abstracts, 5608D043.

24. Liang, L.; Shi, J.; Yejin Fenxi 1993, 13, 45; apud Anal. Abstracts, 5606D012.
25. She, Z. B.; Wang, Z. H.; Fenxi Huaxue 1993, 21, 1313; apud Anal. Abstracts, 5605D045.

26. Chen, Z. H.; Lihua Jianyan Huaxue Fence 1993, 29, 28; apud Anal. Abstracts, 5604D020.

27. Yao, B. H.; Chen, H. Y.; Fang, H. Q.; Fenxi Shiyanshi 1993, 12, 38; apud Anal. Abstracts, 5603H280.

28. Sharma, P.; Kumbhat, S.; Rawat, C.; Int. J. Envirom. Anal. Chem. 1992, 48, 201.

29. Zhang, Z.Q.; Chen, S.Z.; Lin, H.M.; Zhang, H.; Anal. Chim. Acta 1993, 272, 227.

30. Economou, A.; Fielden, P. R.; Analyst 1993, 118, 47.

31. Zhang, Z. Q.; Zhang, S. Z.; Chen, Z. P.; Liu, X. L.; Fenxi Shiyanshi 1992, 11, 21; apud Anal. Abstracts, 5506H190.

32. Paneli, M. G.; Voulgaropoulos, A. N.; Fresenius'-J.Anal. Chem. 1991, 341, 716.

33. Feng, G.; Chen, H.; Li, T.; Fenxi Huaxue 1991, 19, 812; apud Anal. Abstracts, 5407D190.

34. Locatelli, C.; Fagioli, F.; Garai, T.; Anal. Chem. 1991, 63,1409

35. Tan, A.; Fresenius'-J.Anal. Chem. 1991, 339, 895

36. Liang, L.; Shi, J.; Sichuan Daxue Xuebao, Ziram Kexueban 1990, 27, 210

37. Zhang, H.; Wollast, R.; Patriarche, G. J.; Analyst 1989, $114,1597$.

38. Odashima, T.; Kawate, Y.; Ishii, H.; Bunseki Kagaku 1988, 37, 439; apud Anal. Abstracts, 5012D206.

39. Saraswathi, K.; Rao, B. S.; J. Eletrochem. Soc. India 1987, 36, 287.

40. Kurbatov, D. I.; Nikitina, G. A.; Zh. Anal. Khim. 1986, 41, 2009; apud Anal. Abstracts, 4908D040.

41. Adelouju, S. B.; Tran, T.; Anal. Lett. 1986, 19, 1633.

42. Jin, W.; Xu, H.; Fenxi Huaxue 1986, 14, 541; apud Anal. Abstracts, 4904D021.

43. Jin, W.; Jiang, W.; Hou, Y.; Fenxi Huaxue 1986, 14, 253; apud Anal. Abstracts, 4901D049.

44. Sviridenko, V. G.; Lapitskaya, S. K.; Lin, D. G.; Eliseeva, I. M.; Zh. Anal. Khim. 1986, 41, 532; apud Anal. Abstracts, 4811D114.

45. Markova, I. V.; Shirokova, V. I.; Zh. Anal. Khim. 1984, 39, 1841; apud Anal. Abstracts, 4711D220.

46. Adeloju, S. B.; Bond, A. M.; Briggs, M. H.; Anal. Chim. Acta 1984, 164, 181.

47. Ostapczuk, P.; Stoeppler, M.; Nuernberg, H. W.; Fresenius'-J.Anal. Chem. 1984, 317, 252.

48. Budnikov, G. K.; Ulakhovich, V. A.; Postnova, I. V.; J. Eletroanal. Chem. Interfacial Eletrochem. 1983 154, 171.

49. Rao, A. L. J.; Brar, B. S.; Puri, B. K.; Sethi, C. L.; Ann. Chim. (Rome) 1983, 73, 449.

50. Li, M.; Xu, G. Y.; Fenxi Shiyanshi 1996, 15, 24; apud Anal. Abstracts, 5803E013.

51. Garcia Rodriguez, A. M.; Cano Pavon, J.M.; Bosch Ojeda, C.; Garcia de Torres, A.; Mikrochim. Acta 1995, 118, 229.

52. Ren, S. X.; Gao, L.; Anal. Lett. 1995, $28,1665$.

53. Sengupta, J. G.; Bouvier, J.L.; Talanta 1995, 42, 269.

54. Lau, O.W.; Ho, S.Y.; Anal. Chim. Acta 1993, $280,269$.

55. Chen, S. G.; Liu, W.; Wang, Y. H.; Wang, D. X.; Fenxi Shiyanshi 1993, 12, 16; apud Anal. Abstracts, 5606E088.

56. Rigin, V.; Anal. Chim. Acta 1993, 283, 895.

57. Zhang, J.S.; Li, L.H.; Li, B.; Fenxi Huaxue 1993, 21, 1474; apud Anal. Abstracts, 5606D045.

58. Caldera, A.; Gomes, E.; Manuel-Estrela, J.; Cerda, V.; Anal. Chim. Acta; 1993;272,339.

59. Hu, Q.; Xie, C. X.; Yang, S. Z.; Fenxi Huaxue 1992, 20 , 1439; apud Anal. Abstracts, 5509E078.

60. MacLaren, J. W.; Lam, J. W. H.; Berman, S. S/.; Akatsuka, K.; Azeredo, M. A.; J. Anal. At. Spectrom. 1993, 8, 279.

61. Chen, J. S.; Berndt, H.; Toelg, G.; Fresenius'-J.Anal. Chem. 1992, 344, 526. 
62. Saran, R.; Basu-Baul, T.S.; Srinivas, P.; Khathing, D. T.; Anal. Lett. 1992, 25, 1545.

63. Parkhomenko, E. P.; Falendysh, N. F.; Pilipenko, A. T.; J. Water Chem. Technol. (Engl. Transl.) 1991, 12, 59.

64. Shen, Z.; Nie, F.; Chen, Y.; Fenxi Huaxue 1991, 19, 1272; apud Anal. Abstracts, 5410D014.

65. Shi, L.; Li, Z.; Xu, Z.; Pan, Z.; Wang, L.; J. Chemom. 1991, 5, 193.

66. Ni, Y.; Zhang, L.; Fenxi Huaxue 1991, 19, 1205; apud Anal. Abstracts, 5409A018.

67. Shen, Z.; Nie, F.; Chen, Y.; Lihua Jianyan, Huaxue Fence 1991, 27, 273; apud Anal. Abstr. 5408D020.

68. Shen, Z.; Nie, F.; Chen, Y.; Lihua Jianyan, Huaxue Fence 1991, 27, 80; apud Anal. Abstr. 5405D006.

69. Lu, X.; Fenxi Huaxue 1991, 19, 235; apud Anal. Abstracts, 5404D192.

70. Li, Z.; Qiu, X.; Yu, R.; Shi, Y.; Lihua Jianyan, Huaxue Fence 1991, 27, 36; apud Anal. Abstr. 5403F015.

71. Blanco, M.; Coelho, J.; Iturriaga, H.; Maspoch, S.; Riba, J.; Quim. Anal. (Barcelona) 1990, 9,269.

72. Ni, Y.; Fenxi Huaxue 1990, 18, 1138; apud Anal. Abstracts, 5401D152.

73. Kato, K.; Bunseki Kagaku 1990, 39, 439; apud Anal. Abstr. 5309D068.

74. Jin, Y.; Yejin Fenxi 1990, 10, 53; apud Anal. Abstr. 5308D071.

75. Jin, Y.; Fenxi Huaxue 1990, 18, 257; apud Anal. Abstr. 5302D112.

76. Shen, Z.; Chen, Y.; Nie, F.; Fenxi Huaxue 1990, 18, 257; apud Anal. Abstr. 5304D023.

77. Zhao, F.; Feng, J.; Lihua Jianyan, Huaxue Fence 1990, 26, 92; apud Anal. Abstr. 5303D010.

78. Li, Z.; Peng, P.; Li, J.; Fenxi Huaxue 1989, 17, 257; apud Anal. Abstr. 5211B011.

79. Gong, W.; YankuangCeshi 1989, 8, 317; apud Anal. Abstr. 5211B006.

80. Arias, J. J.; Jimenez, A. I.; Jimenez, F.; Mikrochim. Acta 1989, I, 303.

81. Ping, X.; YankuangCeshi 1989, 8, 101; apud Anal. Abstr. 5207B145.

82. Liu, Y.; Chen, M.; Yi, Y.; YankuangCeshi 1989, 8, 17; apud Anal. Abstr. 5109B076.

83. Lu, X.; Shi, W.; Fenxi Shiyanshi 1988, 7, 7; apud Anal. Abstr. 5110B048.

84. Ke, Y.; Yu, S.; Xiao, X.; Fenxi Shiyanshi 1988, 7, 5; apud Anal. Abstr. 5106B033.

85. Cheng, D.; Li, Z.; Lihua Jianyan, Huaxue Fence 1988, 24, 281; apud Anal. Abstr. 5105H083.

86. Lopes, M. H.; Gomes, J. M. C.; Medinilla, J.; Sanches, F. G.; Quim. Anal. (Barcelona) 1988, 7,341.

87. Fang, G.; Zhao, C.; Fenxi Shiyanshi 1988, 7, 12; apud Anal. Abstr. 5105B191.

88. Wang, Z.; Zhou, G.; Luo, G.; Lihua Jianyan, Huaxue Fence 1988, 24, 263; apud Anal. Abstr. 5105B034.
89. Kane, J. S.; J. Anal. Atom. Spectrom. 1988, 3, 1039.

90. Murillo, J. A.; Lemus, J. M.; de la Peña, A. M.; Salinas, F.; Analyst 1988, 113, 1439.

91. Li, J.; Xi, C.; Shi, H.; Fenxi Huaxue 1988, 16, 311; apud Anal. Abstr. 5010B235.

92. Ni, Y.; Fenxi Huaxue 1987, 15, 995; apud Anal. Abstr. 5006B193.

93. Klaentschi, N.; Essenwein, A.; Mueller, T.; Fresenius'J.Anal. Chem. 1987, 328, 657.

94. Ambhore, D. P.;Joshi, A.P.; Indian J. Chem. 1986, 25(A), 699.

95. Luo, S.; Yang, Z.; Fenxi Huaxue 1986, 14, 47; apud Anal. Abstr. 4809B212.

96. Luo, C.; Wu, Y.; Wu, F.; Fenxi Huaxue 1985, 13, 832; apud Anal. Abstr. 4808B143.

97. Rios, A.; Valcarcel, M.; Talanta 1985, 32, 851

98. Vargas, M. G.; Milla, M.; Antequera, I.; Bustamente, J. A. P.; Anal. Chim. Acta 1985, 171, 313.

99. Rao, A. L. J.; Shekhar, C.; Microchem. J. 1984, 30, 283.

100. Nesterenko, P. N.; Ivanov, V. N.; Zh. Anal. Khim. 1983, $38,1415$.

101. Deshmukh, B. K.; Chem. Anal. 1983, 28, 297; apud Anal. Abstr. 4605B214.

102. Wasey, A.; Bansal, R.K.; Satake, M.; Puri, B.K.; Bunseki Kagaku 1983, 32, E211; apud Anal. Abstr. 4602B078.

103. McLaren, J.W.; Berman, S. S.; Boyko, V. J.; Russel, D. S.; Anal. Chem. 1981, 53, 1802.

104. Savinova, E. N.; Korobova, E. M.; Shumskaya, T. V.; Zh. Anal. Khim. 1981, 36, 1267; apud Anal. Abstr..

105. Ilyas, S. Q. R.; Joshi, A. P.; J. Indian Chem. Soc. 1981, 58,360 .

106. Mukherjee, S.; Garg, S. K.; Garg, S. K.; Singh, R. P.; Chem. Ind. (London) 1981, 93.

107. Bruno, P.; Caselli, M.; Gatti, C.; Traini, A.; Anal. Lett. 1980, 13, 1497

108. Maksimova, I. M.; Morosanova, E. I.; Zh. Anal. Khim. 1994, 49, 602; apud Anal. Abstr. 5703D165.

109. Arruda, M. A. Z.; Zagatto, E. A. G.; Maniasso, N.; Anal. Chim. Acta 1993, 283, 476.

110. Ridder, C.; Noergard, L.; Chemom. Intell. Lab. Syst. 1992, 14, 297.

111. Tagashira, S.; Onoue, K.; Murakami, Y; Sasaki, Y.; Anal. Sci. 1992, 8, 307.

112. Yamane, T.; Ishimizu, C.; Mikrochim. Acta 1991, I, 121.

113. Eskilson, H., Haraldsson, C.; Jagner, D.; Anal. Chim. Acta $1985175,79$.

114. Fernandez, A.; Luque de Castro, M. D.; Valcarcel, M.; Anal. Chem. 1984, 56, 1146.

115. Betteridge, D.; Fields, B; Freseniu's Z. Anal. Chem 1983; 314,386

116. Buldini, P. L.; Ferri, D.; Nobili, D.; Eletroanalysis (New York) 1991, 3, 559

117. Department of Environment (UK); Methods Exam. Waters Assoc. Mater. 1987, 144; apud Anal. Abstr. $5101 \mathrm{H} 032$ 